\title{
A New Parameterization of Single Scattering Solar Radiative Properties for Tropical Anvils Using Observed Ice Crystal Size and Shape Distributions
}

\author{
Greg M. McFarquhar \\ Department of Atmospheric Sciences, University of Illinois at Urbana-Champaign, Urbana, Illinois \\ PING YANG \\ Department of Atmospheric Sciences, Texas A\&M University, College Station, Texas \\ ANDREAS MACKE \\ Institut fuer Meereskunde, Universitat zu Kiel, Cologne, Germany \\ ANTHONY J. BARAN \\ Met Office, Bracknell, United Kingdom
}

(Manuscript received 23 May 2001, in final form 21 February 2002)

\begin{abstract}
Parameterizations of single scattering properties currently used in cloud resolving and general circulation models are somewhat limited in that they typically assume the presence of single particle habits, do not adequately account for the numbers of ice crystals with diameters smaller than $100 \mu \mathrm{m}$, and contain no information about the variance of parameterization coefficients. Here, new parameterizations of mean single scattering properties (e.g., single scatter albedo, asymmetry parameter, and extinction efficiency) for distributions of ice crystals in tropical anvils are developed. Using information about the size and shape of ice crystals acquired by a twodimensional cloud probe during the Central Equatorial Pacific Experiment (CEPEX), a self-organized neural network defines shape based on simulations of how the particle maximum dimension and area ratio (ratio of projected area to that of circumscribed circle with maximum dimension) vary for random orientations of different idealized shapes (i.e., columns, bullet rosettes, rough aggregates, and particles represented by Chebyshev polynomials). The size distributions for ice crystals smaller than $100 \mu \mathrm{m}$ are based on parameterizations developed using representative samples of 11633 crystals imaged by a video ice particle sampler (VIPS). The meanscattering properties for distributions of ice crystals are then determined by weighting the single scattering properties of individual ice crystals, determined using an improved geometric ray-tracing method, according to number concentration and scattering cross section.

The featureless nature of the calculated phase function, averaged over all observed sizes and shapes of ice crystals, is similar to that obtained using other schemes designed to account for variations in sizes and shapes of ice crystals. The new parameterizations of single scatter albedo, asymmetry parameter, and extinction efficiency are then determined by functional fits in terms of cloud particle effective radius; there was no statistically significant dependence on either ice water content or temperature. Uncertainty estimates incorporated into the parameterization coefficients are based upon a Monte Carlo approach. Comparisons with previously used parameterizations and with parameterizations developed using single crystal habits are made to show that the determination of representative crystal habits is still a major unknown in the development of parameterization schemes.
\end{abstract}

\section{Introduction}

Ice clouds, especially cirrus, have been shown in general circulation model (GCM) and satellite studies to have a major effect on the earth's radiation balance and

Corresponding author address: Dr. Greg M. McFarquhar, Dept. of Atmospheric Sciences, University of Illinois at Urbana-Champaign, 105 S. Gregory St., Urbana, IL 61801-3070.

E-mail: mcfarq@atmos.uiuc.edu climate as a result of the significant contribution they make to the diabatic heating of the upper troposphere (Ramaswamy and Ramanathan 1989). For example, Zhang et al. (1999) found that modeled radiative fluxes were sensitive to the specification of effective radius $r_{e}$ and that the most realistic vertical distribution of clouds was obtained from the experiment including the most complete representation of cloud microphysics.

An accurate description is needed not only of the cloud microphysics, but also of cloud-radiative inter- 
actions. The weakest physical link for the representation of microphysical and radiative processes in cloud-and larger-scale models are connected descriptions of the microphysical and radiative characteristics. Because of the complex nonlinear interactions between radiation and microphysics, it has been necessary to parameterize or simplify the results of detailed radiative transfer models for incorporation into the cloud- and larger-scale models. Large-scale atmospheric models must account for both the solar and longwave effects of ice crystals. In this study, attention is focused on shortwave radiation.

Takano and Liou (1989, 1995) were among the first to develop a theoretical framework for the computation of solar radiative transfer with application to ice crystals. Using these results and other related studies, many parameterizations for the optical properties of ice crystals in terms of effective radius $\left(r_{e}\right)$, ice water content (IWC), and temperature have been developed. Ebert and Curry (1992, hereafter EC92) parameterized the shortwave radiative properties (i.e., extinction coefficient $\beta_{\text {ext }}$, single scatter albedo $\omega_{0}$, and asymmetry parameter $g$ of hexagonal cylinders for use in climate models). Fu (1996, hereafter F96) then developed an accurate parameterization of solar radiative properties of cirrus clouds using improved light scattering calculations of hexagonal ice crystals. Later, Kristjansson et al. (1999, hereafter K99) developed separate parameterizations for the optical properties of ice crystals for different ice crystal shapes. Other parameterizations include that of Wyser and Yang (1998, hereafter WY98) who computed bulk scattering properties for various size distributions and crystal shapes.

There are differences in the relationships used to parameterize interactions between single scattering radiative properties and cloud microphysical properties because of the different radiative methods used and especially because of the different crystal shapes used to represent the distributions of ice crystals. Given the uncertainty or variability in the occurrence of different habits in ice clouds, and given that clouds almost always consist of mixtures of habits rather than a single habit, such discrepancies should be expected. Further, there may not be universal relations between single scattering properties and microphysical properties that apply to all ice clouds.

Here, parameterizations of mean single scattering properties (e.g., single scatter albedo, extinction coefficient, and asymmetry parameter) are developed for tropical anvils using crystal sizes observed, and shapes derived from data collected during the Central Equatorial Pacific Experiment (CEPEX). These habit-dependent size distributions are combined with an improved geometric ray-tracing method referred to as GOM2 (Yang et al. 2000, hereafter Y00) by weighting the single scattering properties of individually sized and shaped particles by scattering cross section and number concentration to obtain the mean scattering properties.
Improvements over past parameterizations are accomplished by using observed mixtures of crystal shapes and sizes rather than using a single crystal habit such as hexagonal columns, by including contributions from ice crystals with diameters smaller than $100 \mu \mathrm{m}$ in the calculation of the radiative properties, and by including uncertainty estimates on the parameterization coefficients. The small ice crystal distributions are based upon a parameterization of observations of small ice crystals obtained with a video ice particle sampler (VIPS) during CEPEX (McFarquhar and Heymsfield 1997, hereafter MH97), and the uncertainty estimates are based upon a bootstrap approach (Efron and Tibshirani 1993), a modified Monte Carlo technique that is useful when the processes underlying the measurement errors are not well known.

Accurate parameterizations of single scattering parameters are needed in order to accurately estimate shortwave radiative fluxes. For example, Vogelman and Ackerman (1995) showed that to obtain the flux accuracy criteria of $\pm 5 \%$ that they identified, for typical asymmetry parameters of 0.80 , accuracies in asymmetry parameter of better than $2 \%$ and $5 \%$ are needed for optical depths of 12 and 2, respectively.

\section{Microphysical data and processing techniques}

\section{a. Methodology}

During CEPEX, conducted from 7 March to 5 April 1993 out of Nadi, Fiji, approximately 35 h of microphysical measurements were collected in blowoff anvils associated with deep convection using the Aeromet Learjet (e.g., MH97). Large ice crystals with maximum dimensions $\left(D_{\max }\right)$ larger than $100 \mu \mathrm{m}$ were measured by a two-dimensional cloud (2DC) probe. These probes are unreliable for measuring the concentrations of particles smaller than about $100 \mu \mathrm{m}$ (Baumgardner and Korolev 1997) and hence data from the VIPS, obtained at the same time and location as the 2DC measurements, must be used to determine the concentrations of smaller ice crystals. Because the analysis of these data is not automated and is time consuming, only a representative sample of 11633 images has been analyzed. MH97 have developed a parameterization of the size distribution of small ice crystals in terms of total ice water content based upon this sample. In this section, the methodology for obtaining size and shape distributions covering the entire size range of ice crystals is reviewed.

\section{b. Large crystal size/shape distributions}

Habit-dependent size distributions were calculated for all times when good quality 2DC data were recorded during CEPEX using an averaging period of $10 \mathrm{~s}$. Each particle measured by the $2 \mathrm{DC}$ is characterized by a maximum dimension and area ratio, which is the ratio of particle cross-sectional area to that of a circumscribed 
TABLE 1. Principle axis length and eigenvectors describing the covariance matrices for the $(a, b)$ and $(c, m)$ fit coefficients in Eqs. (1) and (2). Because coefficients of Eq. (1) is obtained by performing a linear fit of $\log \left(\operatorname{IWC}_{<100}\right)$ vs $\log \left(\mathrm{IWC}_{T}\right)$, the first parameter is a $\operatorname{logarithm}$ of $a$.

\begin{tabular}{|c|c|c|c|c|}
\hline Parameter & Units & Axis length & Eigenvector & Best value \\
\hline $\log (a)$ Eq. (1) & $a\left(\mathrm{~g} \mathrm{~m}^{-3}\right)$ & 0.378016 & $(0.914265,-0.405117)$ & -0.599 \\
\hline$b$ Eq. (1) & Dimensionless & 0.0471792 & $(0.405117,0.914265)$ & 0.837 \\
\hline$m$ Eq. (2) & $\mu \mathrm{m}^{-1}$ & 0.00438119 & $(0.376274,0.926508)$ & -0.0494 \\
\hline
\end{tabular}

circle. Following the approach of McFarquhar et al. (1999, hereafter M99) a habit was assigned to each crystal imaged using a self-organized neural network scheme which determines shape based on simulations of how the maximum particle dimension and area ratio vary for random orientations of different idealized shapes (i.e., columns, bullet rosettes, and rough aggregates, the latter being different than the Koch fractal polycrystals used by M99). The maximum dimension measured by the $2 \mathrm{DC}$ is converted to particle maximum dimension by accounting for the manner in which the idealized crystals would be sized by the discrete photodiode array (M99). The mass of each idealized particle is determined from the volume and density of the idealized particle. Because the single scattering properties needed for calculating the radiative properties are only available for a number of discrete sizes, the habit-dependent size distributions are generated for bins centered at the following maximum dimensions: 100, 130, $175,225,275,350,450,550,650,750,900,1150,1400$, 1750,2500 , and $3500 \mu \mathrm{m}$.

\section{c. Small crystal size/shape distributions}

The use of the MH97 small crystal parameterization allows accurate information about small crystals to be included. MH97 parameterized the ice water content contained in particles with melted equivalent diameters $D_{m}$ $<100 \mu \mathrm{m}\left(\mathrm{IWC}_{<100}\right)$ as a function of the ice water content in all particle sizes $\mathrm{IWC}_{T}$ based on observations as

$$
\mathrm{IWC}_{<100}=\min \left[\mathrm{IWC}_{T}, a\left(\frac{\mathrm{IWC}_{T}}{\mathrm{IWC}_{0}}\right)^{b}\right],
$$

where $\mathrm{IWC}_{T}=\mathrm{IWC}_{<100}+\mathrm{IWC}_{>100}, \mathrm{IWC}_{>100}$ is the ice water content in particles with $D_{m}>100 \mu \mathrm{m}$, and $\mathrm{IWC}_{0}$ $=1 \mathrm{~g} \mathrm{~m}^{-3}$. Because only $\mathrm{IWC}_{>100}$, as measured by the 2DC is known, the solution of MH97's parameterization for IWC $_{<100}$ requires the use of Newton's algorithm, a root-finding scheme. Following MH97 the slope of a firstorder gamma function $\left(\alpha_{<100}\right)$ characterizing the small crystals as a function of IWC $_{<100}$ was expressed as

$$
\alpha_{<100}=c-m \log _{10}\left(\frac{\mathrm{IWC}_{<100}}{\mathrm{IWC}_{0}}\right),
$$

where the units of $a, b, c$, and $m$ are included in Table 1 .

Possible values for the model parameters are obtained by using the bootstrap method to generate a constant chi-squared boundary in the phase space of model parameters [e.g., $(a, b)$ or $(c, m)$ phase space]. As an example, the calculation of a confidence region for model parameters in $(a, b)$ phase space is illustrated. First, the most likely values of $a$ and $b\left(a_{\text {best }}, b_{\text {best }}\right)$ are determined minimizing chi-squared expressed by

$$
\chi^{2}(a, b)=\sum_{i=1}^{N} \frac{y_{i}-y\left[x_{i} ;(a, b)\right]}{\sigma_{i}},
$$

where $y_{i}$ represents $\mathrm{IWC}_{<100}, x_{i}$ represents $\mathrm{IWC}_{T}, N$ is the total number of data points, $y$ is the function given by Eq. (1), and the weighting $\sigma_{i}$ of all the data points is equal. The chi-squared for the best fit is represented as $\chi_{\min }^{2}$. The boundaries of a surface with center at $\left(a_{\text {best }}\right.$, $\left.b_{\text {best }}\right)$ is then constructed by determining a constant chisquared boundary around this region (Press et al. 1992, p. 688), with $\chi^{2}=\chi_{\min }^{2}+\Delta \chi^{2}$. The boundaries of this surface are given by an ellipse in two-dimensional phase space. An orthonormal set of vectors $\mathbf{V}$, which describes the principle axes of the constant chi-squared ellipse, can be generated from the eigenvectors and eigenvalues of the inverse of the covariance matrix obtained for the fit. The length of the ellipse axis are determined from the eigenvalues and $\Delta \chi^{2}$.

The value of $\Delta \chi^{2}$ is estimated using the bootstrap approach. Five hundred different realizations of the $\left(\mathrm{IWC}_{<100}, \mathrm{IWC}_{T}\right.$ ) pairs measured by MH97 were obtained by randomly drawing data points, with replacement, from the original dataset. By performing the fits for each realization, not only is a library of possible $a$ and $b$ values calculated, but also the standard deviation of the chi-squared values describing the goodness of the fit. The later value represents $\Delta \chi^{2}$. Figure 1 shows the values of $a$ and $b$ generated from the bootstrap approach, together with the constant chi-squared boundary, in $(a$, $b$ ) phase space. There is considerable variation about the $\left(a_{\text {best }}, b_{\text {best }}\right)$ value that occurs because of the large amount of scatter in the relation between $\mathrm{IWC}_{<100}$ and $\mathrm{IWC}_{T}$. The curve plotted is not a true ellipse because the constant chi-square ellipse boundary was derived in logarithmic space, but plotted in regular coordinates. Note also that independently choosing a value for the $a$ coefficient in the range $a_{\text {best }} \pm \Delta a$ and for $b$ in the range $b_{\text {best }} \pm \Delta b$ would give $(a, b)$ pairs that are not within the range of possible solutions.

Figure 2 illustrates that the deviations in the $(a, b)$ coefficients must be accounted for in the application of Eq. (1). The different grey lines represent the parame- 


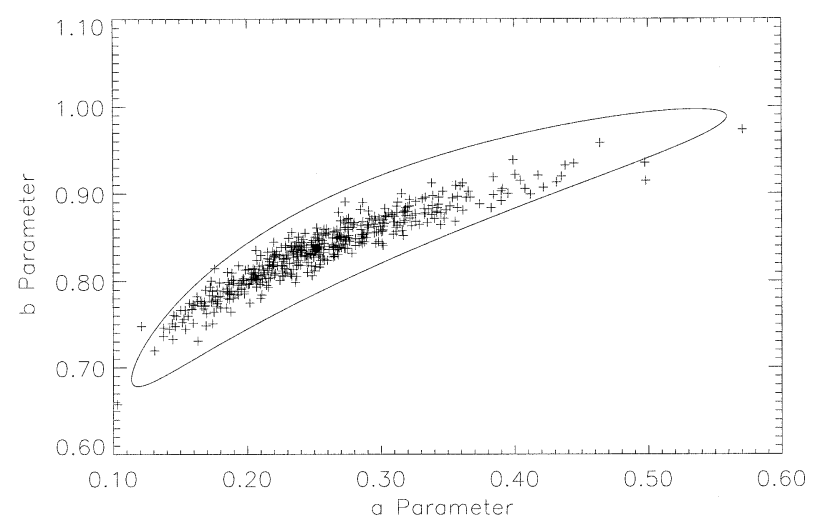

FIG. 1. Possible solutions for $a$ and $b$ parameter describing relation between $\mathrm{IWC}_{T}$ and $\mathrm{IWC}_{<100}$. Pluses represent possible values obtained by applying the bootstrap approach to data used by MH97 to develop original fit. The solid line represents the surface-defining constant chi-squared boundary, where all values inside represent equally realizable values of the $(a, b)$ coefficients.

terized relation between $\mathrm{IWC}_{<100}$ and $\mathrm{IWC}_{T}$ obtained by randomly choosing $a$ and $b$ coefficients within the constant chi-squared boundary, whereas the thick line represents the relation deduced from the most likely values of $a$ and $b$. Substantial deviation from that line described by Eq. (1) is noted. Some data points still lie outside of the range of possible solutions, even when the variances are accounted for. This suggests that Eq. (1) might not adequately characterize the relationship between $\mathrm{IWC}_{<100}$ and $\mathrm{IWC}_{T}$, or that dependences on other parameters should be considered. At this time there are not sufficient data available to determine better relationships.

A similar procedure was followed to derive the phase space of possible solutions for the $c$ and $m$ coefficients in Eq. (2). The properties of the ellipses that describe the constant chi-squared boundaries in $(a, b)$ and $(c, m)$ phase space are summarized in Table 1, with the lengths of the principle axis, the orthonormal vectors describing the principle axis, and the most likely values of the $a$, $b, c$, and $m$ coefficients listed.

The data used to generate Eq. (1) were obtained when total IWCs were less than $0.1 \mathrm{~g} \mathrm{~m}^{-3}$ because the VIPS became saturated at higher IWCs. Because IWCs between 0.1 and $1.0 \mathrm{~g} \mathrm{~m}^{-3}$ frequently occur in tropical anvils, it is necessary to extrapolate to conditions of higher IWCs in order to apply these fits in climate and cloud resolving models. When extrapolating, Eq. (1) shows that crystals with $D_{\max }$ less than $100 \mu \mathrm{m}$ make smaller fractional contributions to IWC as total IWC increases. Using forward scattering spectrometer data (FSSP) as an upper bound for the masses of smaller crystals, McFarquhar and Heymsfield (1996, hereafter MH96) also showed that the fractional mass and fractional optical depth contained in small crystal sizes decreased at higher IWCs.

There is considerable controversy as to what constitutes representative concentrations of small ice crystals

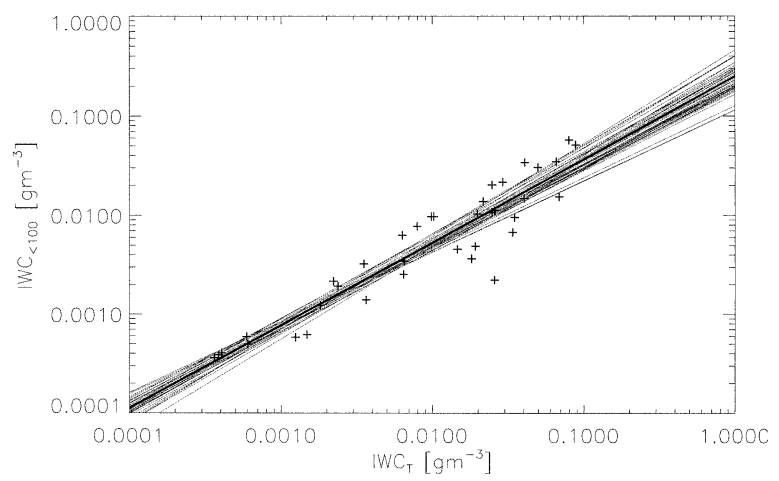

FIG. 2. Relationship between $\mathrm{IWC}_{<100}$ and $\mathrm{IWC}_{T}$. Pluses represent original data points obtained by MH97. Solid lines represent different representations of the relationship between $\mathrm{IWC}_{<100}$ and $\mathrm{IWC}_{T}$ obtained by randomly chosing $(a, b)$ values from the surface of physically realizable solutions. Note that some data points still fall outside the range of these curves.

in the Tropics and even how to measure small crystals. Knollenberg et al. (1993) found very high concentrations of crystals with diameters smaller than $100 \mu \mathrm{m}$ and mass modes in the size range $20-40 \mu \mathrm{m}$ at the tops of cirrus associated with tropical cyclones; however, the spectrometers used to make these measurements may be suspect for $D<30 \mu \mathrm{m}$. Recent studies (e.g., Arnott et al. 2000) suggest that ice particle replicators, which are not too unlike the VIPS in regards to issues of collection efficiency, may underestimate the concentrations of ice crystals with $D<50 \mu \mathrm{m}$; they further suggest that data from an FSSP may provide adequate concentrations when the numbers of larger crystals is low enough. Still other studies (e.g., Gardiner and Hallett 1985) have questioned the utility of the FSSP measurements in ice clouds, especially in the presence of larger ice crystals, the conditions observed during CEPEX. Independent measurements from another instrument are needed to resolve these discrepancies. Because of uncertainties in the measurements of small crystals, a sensitivity study is performed where it is assumed that the FSSP provides adequate measurements of small ice crystals; this provides an upper bound for the maximum possible effect that the small crystals may have on anvil radiative properties. Another sensitivity study limits the mass of small crystals to $\mathrm{IWC}_{<100}=a(0.1)^{b}$, with $a$ and $b$ randomly chosen using the values in Table 1 . This represents a probable lower limit for masses of smaller crystals; there are large uncertainties in extrapolating Eq. (1) to ice water contents larger than $0.1 \mathrm{~g} \mathrm{~m}^{-3}$.

Figures 22 and 23 from MH96 show that many crystals with diameters smaller than $100 \mu \mathrm{m}$ are quasi-circular, meaning that they have area ratios close to unity, but are not exactly circular in shape. In fact, of the 11 633 VIPS particles that were processed, over $90 \%$ were manually identified as spherical or quasi-spherical. Hence, in previous studies (e.g., M99) small ice crystals were represented by spheres. However, some form of deformed sphere may provide a better representation. 
TABLE 2. Maximum dimension and average area ratio for 11633 particles measured by the VIPS during CEPEX. Calculated deformation parameter and volume for particles represented by eighthorder Chebyshev polynomial, conserving maximum dimension and area ratio, are also listed.

\begin{tabular}{cccc}
\hline \hline $\begin{array}{c}\text { Diameter } \\
(\mu \mathrm{m})\end{array}$ & Area ratio & $\epsilon$ & $\begin{array}{c}\text { Volume } \\
\left(\mu \mathrm{m}^{3}\right)\end{array}$ \\
\hline $0-10$ & 0.748 & 0.164 & 42.1 \\
$10-20$ & 0.706 & 0.202 & 1038 \\
$20-30$ & 0.690 & 0.218 & 4631 \\
$30-40$ & 0.692 & 0.216 & $1.28 \times 10^{4}$ \\
$40-50$ & 0.724 & 0.186 & $2.91 \times 10^{4}$ \\
$50-60$ & 0.748 & 0.164 & $5.60 \times 10^{4}$ \\
$60-70$ & 0.753 & 0.160 & $9.34 \times 10^{5}$ \\
$70-80$ & 0.730 & 0.180 & $1.36 \times 10^{5}$ \\
$80-90$ & 0.757 & 0.157 & $2.10 \times 10^{5}$ \\
$90-100$ & 0.752 & 0.161 & $2.90 \times 10^{5}$ \\
\hline
\end{tabular}

Table 2 shows that the observed average area ratio, which should be unity for circular particles, is 0.73 when averaged over all shapes and sizes of small ice crystals. Because extinction coefficient is proportional to projected area, assuming spherical particles would lead to a $37 \%$ error in extinction optical depth. Therefore, a representation was sought that would preserve the quasispherical shape of the particle while allowing for minor departures from a spherical shape. Yang et al. (2001) have previously shown that the top of the atmosphere radiance is sensitive to the shape of ice crystals smaller than $50 \mu \mathrm{m}$. Because of the important role of small ice crystals in atmospheric radiative transfer, it is necessary to include these small ice crystals by using a more realistic shape rather than the spherical approximation in the parameterization of cirrus radiative properties.

Several such shapes were used. Mugnai and Wiscombe (1980) and others have investigated the description of rotationally symmetric scatters by means of Chebyshev particles, which are obtained by continuously deforming a sphere by means of Chebyshev polynomials. Their shape in a spherical coordinate system is given by

$$
r(\theta, \phi)=r_{0}\left[1+\epsilon T_{n}(\cos \theta)\right], \quad|\epsilon|<1,
$$

where $r_{0}$ is the radius of the unperturbed sphere, $\epsilon$ is the deformation parameter, and $T_{n}(\cos \theta)=\cos n \theta$ is the Chebyshev polynomial of degree $n$. Idealized crystals can be represented by a Chebyshev particle of a single degree or as a sum of Chebyshev polynomials. Figure 3 shows the projection of two idealized ice crystals, represented by Chebyshev polynomials, in a two-dimensional plane. For the particle on the left, an eighth degree polynomial is used with $\epsilon$ chosen so that the area ratio of the idealized particle is equal to that of observed particles. Note that this particle is predominantly spherical in nature, even though it may appear planar at this viewing angle. The particle on the right is obtained from a sum of Chebyshev polynomials. Chuang and Beard (1990, hereafter CB90) represented drop shapes by 10th-order Chebyshev polynomials, where the expan-
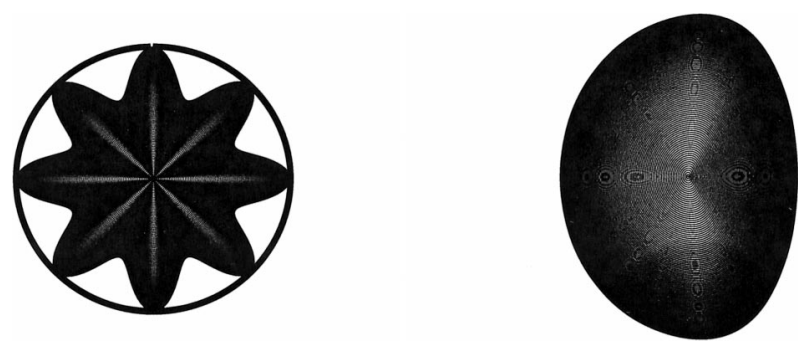

FIG. 3. Different representations for the shapes of ice crystals with dimensions smaller than $100 \mu \mathrm{m}$. (a) A Chebyshev particle with nonzero eighth-degree terms; (b) 10th-order Chebyshev polynomials, with expansion coefficients derived by CB90. The projected area of both crystals equals the average projected area of crystals imaged by the VIPS during CEPEX.

sion coefficients for the different terms are determined from an analysis of equilibrium raindrop shapes. The raindrop shape with area ratio closest to the average area ratio observed for different sizes of small ice crystal is selected and the expansion coefficients given by CB90 are used to describe the small ice crystal shape with appropriate scaling.

\section{d. Composite size/shape distributions}

To account for the stochastic nature of the small crystal parameterization, size- and shape-dependent distributions covering the entire size range are derived as follows. For each of the 11769 2DC distributions, the number concentrations of different sizes and shapes of large crystals are known. The IWC in these large crystal sizes is calculated and used to determine the IWC in smaller crystal sizes and the slope of the first-order gamma distribution, using $(a, b)$ and $(c, m)$ values randomly chosen from the surface of possible solutions (Table 1). The calculated small size distribution is then added to the 2DC observed distribution. The effect of truncating the first-order gamma distribution describing the small crystals at $100 \mu \mathrm{m}$ is accounted for by multiplying all crystal numbers by a constant to ensure that the mass in the small crystal sizes is equal to $\mathrm{IWC}_{<100}$.

Two different sets of distributions are generated, with the small crystal shapes determined using the eighthorder Chebyshev polynomial and the CB90 10th-order expansion coefficients; the large crystal distributions are identical in each set of distributions. Henceforth, the eighth-order Chebyshev polynomials will represent the base distributions and the distributions generated with the CB90 coefficients will be used for sensitivity studies.

\section{e. Bulk cloud properties}

To develop the parameterizations in this study, bulk microphysical properties such as IWC and $r_{e}$ need to be calculated for all of the distributions. IWC is defined by

$$
\mathrm{IWC}=\sum_{i=1} \sum_{j=1} n\left(D_{i, j}\right) M\left(D_{i, j}\right)
$$


where $D_{i, j}$ is the maximum dimension of particles in size bin $i$ and habit bin $j, n\left(D_{i, j}\right)$ is the number concentration, and $M\left(D_{i, j}\right)$ is the corresponding mass. The definition of effective radius is not as straightforward. McFarquhar and Heymsfield (1998) reviewed several definitions of $r_{e}$ that have been used by different authors. They concluded that the use of the F96 or Foot (1988) definition is most desirable because it is proportional to the ratio between ice mass content and cross-sectional area, whereas many other definitions are not. Definitions that rely on either equivalent-area or equivalent-volume particles provide less physical insight since the radiative properties of such particles differ more from those of particles which conserve both volume and surface area. Thus, $r_{e}$ and effective diameter $\left(D_{e}=2 r_{e}\right)$ are defined according to F96 as

$$
r_{e}=\frac{\sqrt{3}}{3 \rho_{i}} \frac{\mathrm{IWC}}{A_{c}},
$$

where $\rho_{i}$ is $0.91 \mathrm{~g} \mathrm{~cm}^{-3}$ and represents the bulk density of ice, and $A_{c}$ is the cumulative cross-sectional area of the ice crystal size distribution.

\section{Derivation of mean single scattering properties}

To calculate the mean single scattering properties of distributions of ice crystals, the size and shape information from the derived size and shape distributions is combined with radiative properties calculated for specific shapes and sizes of ice crystals. Y00 describe GOM2, which is used to provide the radiative properties of specific shapes and sizes of ice crystals for the 56 different visible and near-infrared wavelengths listed in Table 1 of Y00. For size parameters on the order of 1520 , this method provides more accurate results than the conventional ray-by-ray Monte Carlo tracing technique that has previously been used to calculate scattering properties. The calculated radiative properties assume that the crystals are randomly oriented in space. The GOM2 library of calculated radiative properties is available for the following shapes: rough and smooth aggregates, hexagonal columns and plates, bullet rosettes, and dendrites.

Calculated radiative properties for deformed spheres are not available in this library. Hence, the single scattering properties are calculated by means of the raytracing method developed by Macke and Grossklaus (1998). Previous studies have verified that such routines accurately calculate the single scattering properties and scattering phase functions for the wavelengths and particle sizes under consideration. The small ice crystals are randomly oriented in three-dimensional space when calculating radiative properties. This assumption may be inappropriate for crystals with larger aspect ratios, but given the quasi-spherical shape of the smaller crystals and their relatively small aspect ratio this assumption is reasonable. Since radiative properties differ for crystals with preferred and random orientations, the pa- rameterizations developed are dependent upon this orientation assumption (e.g., Baran et al. 2001a).

To combine the scattering properties for different sizes and shapes of ice crystals, the scattering properties of individual ice crystals are weighted by scattering cross section and number concentration following the techniques used by M99, Y00, and others. The scattering properties are computed for the 56 different wavelengths, and then combined into the four broad bands used by CCM3 (0.25-0.69, 0.69-1.19, 1.19-2.38, and 2.38-4.0 $\mu \mathrm{m})$ and other climate models, weighted according to the fraction of solar irradiance at the top of the atmosphere in each band. The wavelength-dependent single scattering albedo is expressed as

$$
\omega_{0}=\frac{\sum_{i} \sum_{j} \beta_{s}\left(D_{i, j}\right) n\left(D_{i, j}\right)}{\sum_{i} \sum_{j} \beta_{e}\left(D_{i, j}\right) n\left(D_{i, j}\right)},
$$

where $\beta_{s}$ is the scattering cross section, and $\beta_{e}$ is the extinction cross section. The scattering phase function is expressed as

$$
\bar{P}(\theta)=\frac{\sum_{i} \sum_{j} \beta_{s}\left(D_{i, j}\right) P\left(\theta, D_{i, j}\right) n\left(D_{i, j}\right)}{\sum_{i} \sum_{j} \beta_{s}\left(D_{i, j}\right) n\left(D_{i, j}\right)},
$$

where $\bar{P}(\theta)$ is the scattering phase function for the ice crystal distribution, and $P\left(\theta, D_{i, j}\right)$ is the scattering phase function for particles with size and shape $D_{i, j}$. Equations similar to Eq. (8) are introduced to give the mean asymmetry parameter $(\mathrm{g})$, and extinction efficiency $\left(Q_{\text {ext }}\right)$. Here, $Q_{\text {ext }}$ is defined as the fractional area of the incident beam removed by the combination of scattering and absorption (extinction).

Sensitivity studies are performed to test the dependences of the calculated single scattering properties on various assumptions. For example, simulations are conducted using Mie theory to calculate the radiative properties of small ice crystals. The Bohren-Huffman Mie scattering routine for calculating scattering and absorption by a homogeneous isotropic sphere is used for this purpose (Bohren and Huffman 1983). Other sensitivity studies are conducted using libraries of phase functions and single scattering properties generated using techniques developed by Macke et al. (1996, hereafter M96) and Baran et al. (2001b, hereafter B01). Both these techniques were not designed to simulate the scattering behavior of individual ice crystals, but rather were designed to represent the scattering behavior of merged size and habit distributions of ice crystals. M96 approximated the highly complex geometric structure of polycrystals observed in cirrus clouds by means of distorted three-dimensional Koch fractal crystals. Although this geometry does not resemble crystals observed in situ, many remote sensing studies have suggested its smoother phase functions and modeled radiative properties are more representative of observed cloud radiative properties than those obtained using single pristine 
(a)

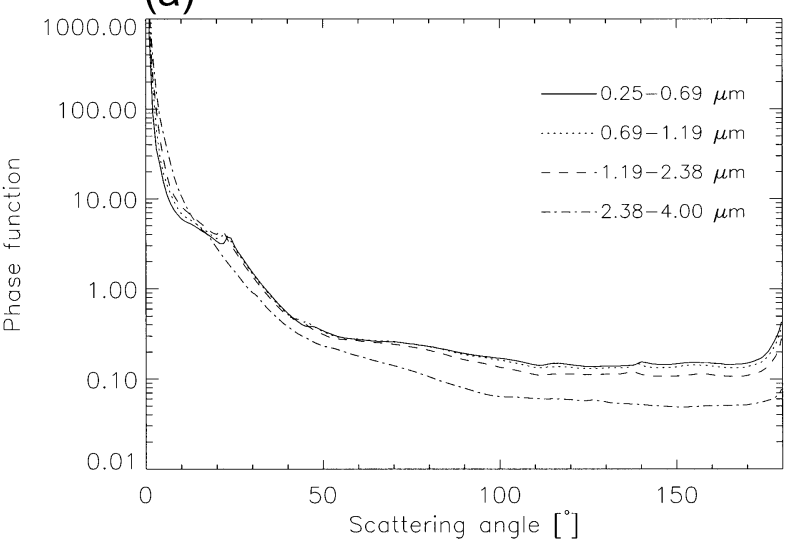

(b)

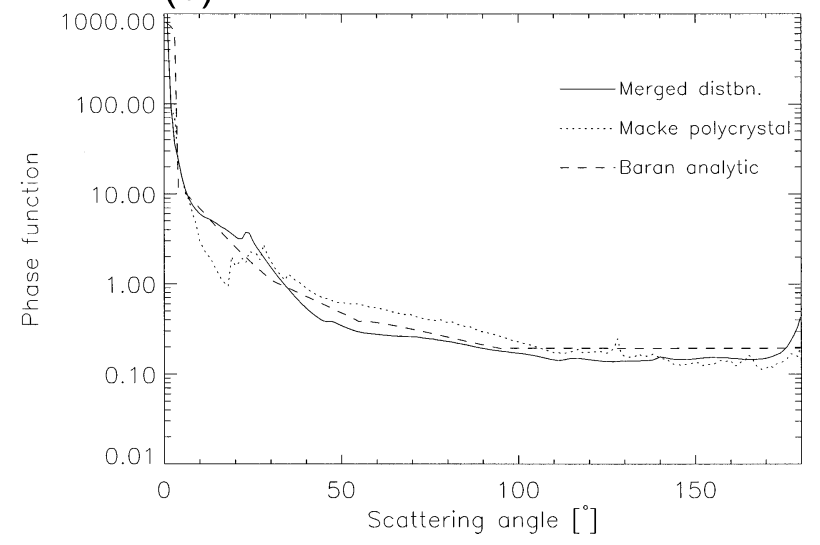

FIG. 4. (a) Normalized mean-scattering phase function obtained by averaging phase functions calculated for all composite size distributions. Different line types represent different wavelength bands. Note the featureless nature of the phase function. (b) Comparison of averaged phase function from (a) for 0.25-0.69- $\mu \mathrm{m}$ band against B01's analytic phase function for same band and M96's phase function for secondorder distorted Koch fractal crystals for $0.664 \mu \mathrm{m}$.

habits (Francis et al. 1999; Baran et al. 1999). The scattering properties of various sizes of second-order distorted Koch fractal polycrystals are calculated at five solar and near-infrared wavelengths $(0.664,0.875$, $1.621,2.142$, and $3.725 \mu \mathrm{m}$ ) using a routine [developed by A. Macke (1996, personal communication)] that calculates the reflection and transmission processes of a bundle of parallel and equidistant incoming rays to the particle, with diffraction at the crystal's geometric cross section computed using the far-field approximation.

B01 have developed an analytic phase function based on a series of simple trigonometric terms, its angular behavior modeled by a laboratory-derived phase function (Volkovitskiy et al. 1980), based on cloud chamber scattering measurements made at visible wavelengths from a collection of nonspherical ice crystals. The point of the analytic phase function is that it captures the essential behavior of scattering from a collection of nonspherical ice crystals, which is an essentially featureless and flat (at scattering angles greater than $90^{\circ}$ ) phase function. The analytic phase function is generated from the asymmetry parameter and can be applied to cirrus clouds for both nonabsorbing and absorbing wavelengths. Using aircraft data at absorbing and nonabsorbing wavelengths, between the scattering angles of about $10^{\circ}$ to $120^{\circ}, \mathrm{B} 01$ found that the analytic phase function better describes the scattered transmitted radiances better than the standard single crystal models (i.e., columns, plates, and rosettes) and the polycrystal model. Further, using Polarization and Directionality of the Earth's Reflectances (POLDER) data from 9000 midlatitude cirrus cases for scattering angles between $60^{\circ}$ and $180^{\circ}$, the analytic phase function minimized differences between modeled phase function and the retrieved spherical albedo. A library of single scattering properties for the same 56 wavelengths and 24 sizes used by $\mathrm{Y} 00$ has been generated to describe this analytic phase function. The mean scattering properties for the distributions of ice crystals are calculated by weighting the scattering properties for individual ice crystals by number concentration and scattering cross section for both the M96 and B01 models.

Figure $4 \mathrm{a}$ shows the normalized mean-scattering phase function averaged over all the CEPEX crystal distributions for the wavelength intervals $0.25-0.69$, $0.69-1.19,1.19-2.38$, and $2.38-4.0 \mu \mathrm{m}$. When compared against scattering phase functions for pristine crystals (e.g., bullet rosettes, hexagonal columns, aggregates), the smooth featureless nature of the merged phase function and the virtual absence of the $22^{\circ}$ and $46^{\circ}$ halos is noticeable. This occurs because small-scale features associated with individual crystals are removed when averaging over the wide range of shapes and sizes making up the size distribution and because Chebyshev polynomials, that are used to characterize the small crystals rather than hexagonal columns or plates, do not have sharp features in their phase functions; random particle orientation also contributes to the smoothing of the phase function. When compared against the phase functions presented by M99, who used spheres to describe the small crystals, the rainbow region due to the sphere assumption has now been removed, probably due to the impact of the Chebyshev particles that appear flatter in that region.

The averaged mean-scattering phase functions generated with the CEPEX data are compared with B01's analytic phase function for the $0.25-0.69 \mu \mathrm{m}$ wavelength and with a phase function calculated for distorted Koch fractal crystals at $0.664 \mu$ m wavelength (M96) in Fig. 4b. There is a pronounced backscatter peak in the merged phase function not visible in the other phase functions. This occurs due to the contributions from the pristine habits that exhibit this backscatter. The complicated structure of the polycrystal causes considerable 


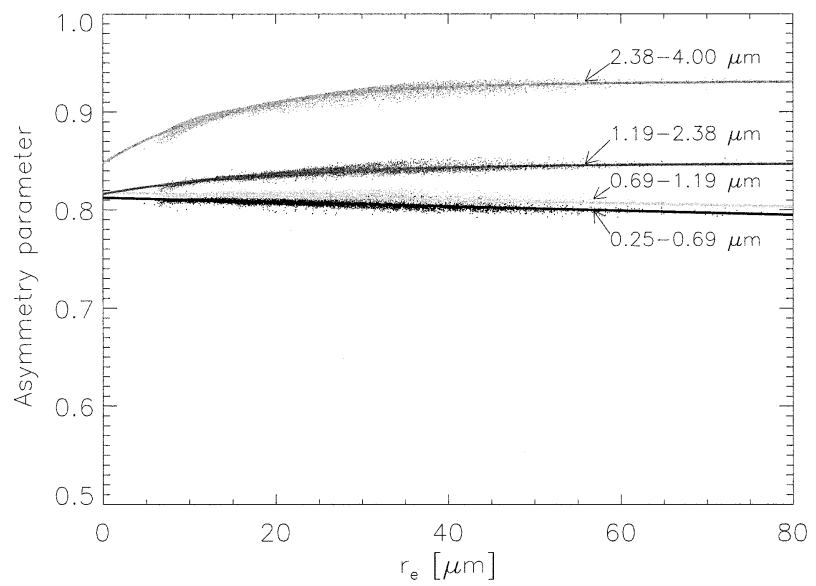

FIG. 5. Asymmetry parameter as function of $r_{e}$ for four different wavelength bands. Dots represent values obtained for all the composite size distributions obtained by combining optical array probe data with small crystal parameterization. Solid lines represent best fit to the data obtained following the method described in text.

deviations of the photons, and the combination of base functions in B01's analytic function is so selective that smooth backscatter is generated. However, the averaged phase function is very similar to the other two in that all are rather featureless.

\section{Parameterization equations}

Following the techniques discussed in section 3 , the single scattering properties are computed for each of the composite crystal distributions derived following the approach outlined in section 2. Investigations were then performed where the dependence of the single scattering properties on $r_{e}$, IWC, and temperature was examined; it was determined that the single scattering properties $g$, $Q_{\text {ext }}$, and $\omega_{0}$ did not depend on IWC and temperature in a statistically meaningful manner. Fits of $g, \omega_{0}$, and $Q_{\text {ext }}$ as functions of $r_{e}$ were then performed using several different relationships. When functional relationships used by other investigators adequately fit the data, such relations were preferred to increase compatibility with previous studies.

Figure 5 plots the asymmetry parameter as a function of $r_{e}$ for the four different wavelength bands considered in Fig. 4a using the base calculations of radiative properties. The scatter in the calculated asymmetry parameters occurs due to variation in the mixture of different habits making up the crystal distributions and due to the variation in the fractional contribution of the small crystals because of the random application of MH97. Additional plots (not shown) showed that the scatter would not be reduced by stratifying the data according to IWC or temperature.

To determine a functional representation characterizing the $g-r_{e}$ relationship, the data are stored into bins with 100 elements in order of increasing $r_{e}$. The difference between the minimum and maximum asymmetry param- eter within each bin provides the uncertainty for each bin. A first order polynomial provided an adequate fit for the first two bands (i.e., 0.25-0.69 and 0.69-1.19 $\mu \mathrm{m}$ ) whereas an exponential function was needed to describe the behavior for the last two bands (i.e., 1.19-2.38 and $2.38-4.0 \mu \mathrm{m})$. This is given by

$$
g= \begin{cases}a_{i}+b_{i} r_{e}, & i=1,2 \\ a_{i}+b_{i} \exp \left(-c_{i} r_{e}\right), & i=3,4,\end{cases}
$$

where the coefficients $a_{i}, b_{i}$, and $c_{i}$ are wavelength dependent; assume that $r_{e}$ is expressed in $\mu \mathrm{m}$. Note that $c_{i}=0$ for $i=1$ or 2 . Separate functions are needed for the different bands because convergence was not obtained for fits of the exponential function to the first two bands and an exponential is required to characterize the decrease in $g$ for small $r_{e}$ for higher bands. There was never any need to use more free parameters to fit the data because uncertainties in any other terms would be greater than the magnitudes of the terms.

As in section $2 \mathrm{c}$, the phase space of possible solutions for the $a_{i}, b_{i}$, and $c_{i}$ parameters is determined from the eigenvalues and eigenvectors of the inverse of the covariance matrix. In order to specify a boundary that would encompass almost all possible solutions, the $\Delta \chi^{2}$ for the boundary was chosen to encompass a $99 \%$ confidence interval $\left(\Delta \chi^{2}=9.21\right.$ in two dimensions and $\Delta \chi^{2}=11.3$ in three dimensions). The surface of possible solutions is hence characterized by an ellipse in two-dimensional $a_{i} / b_{i}$ phase space and an ellipsoid in three-dimensional $a_{i} / b_{i} / c_{i}$ phase space. Table 3 lists the lengths of the principal axis of the ellipse/ellipsoids, the orthonormal vectors describing the principal axis, and the most likely values of the coefficients.

Similar procedures were followed to quantify the relationship between $\omega_{0}$ and $r_{e}$. Figure 6 shows that, again, a single dependence of $\omega_{0}$ on $r_{e}$ characterized the data. First-order polynomials delineated relationships for the first- and second-wavelength bands, whereas the exponential functions were needed to represent the relationships for the higher wavelengths. The fits are represented by

$$
\omega_{0}= \begin{cases}d_{i}+e_{i} r_{e}, & i=1,2 \\ d_{i}+e_{i} \exp \left(-f_{i} r_{e}\right), & i=3,4,\end{cases}
$$

where the coefficients $d_{i}, e_{i}$, and $f_{i}$ are wavelength dependent. Table 4 lists their most likely values, and the properties of the ellipses or ellipsoids describing the region of acceptable solutions, and Fig. 6 shows the fit to the data. Figure 7 and Table 5 define the relationship between $Q_{\text {ext }}$ and $r_{e}$, which is represented by

$$
Q_{\text {ext }}=g_{i}+h_{i} \exp \left(-m_{i} r_{e}\right) \text {. }
$$

Note that because the Macke and Grossklaus (1998) routines simply assume $Q_{\text {ext }}=2$, calculations with Mie theory are used to determine the $Q_{\text {ext }}$ values plotted in this figure.

To describe cloud-radiative interactions, the relation- 


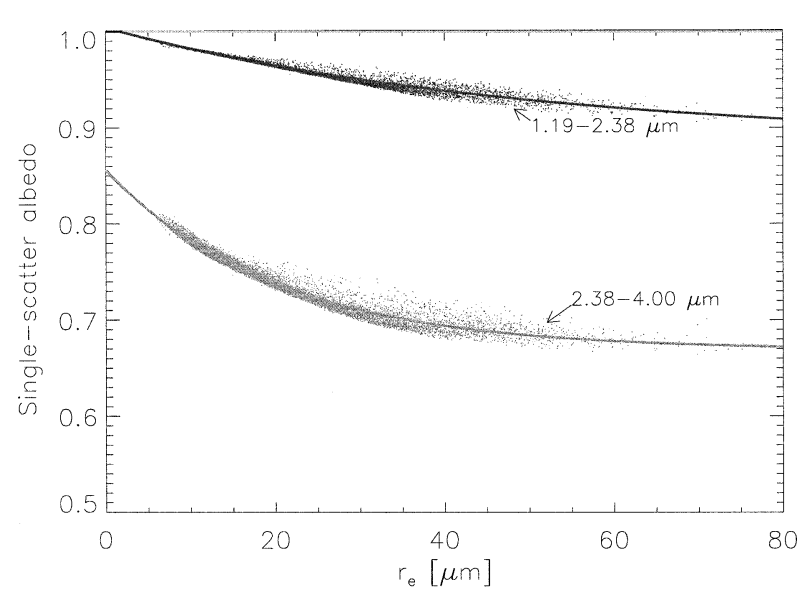

FIG. 6. As in Fig. 5, except for single scatter albedo as a function of $r_{e}$.

ship between extinction coefficient, $\beta_{\text {ext }}$, IWC, and $r_{e}$ is also needed. EC92, WY98, and others have used relationships of the form

$$
\beta_{\text {ext }}=\operatorname{IWC}\left(a_{0}+a_{1} / r_{e}\right)
$$

to characterize these interactions, where $a_{0}$ and $a_{1}$ are wavelength-dependent coefficients. However, given the use of Eq. (6) to define $r_{e}$, a simple expression for $\beta_{\text {ext }}$ is derived as

$$
\beta_{\mathrm{ext}}=\frac{Q_{\mathrm{ext}} \sqrt{3} \mathrm{IWC}}{3 \rho_{i} r_{e}},
$$

where $Q_{\text {ext }}$ is defined by Eq. (11) and depends on $r_{e}$, and $\rho_{i}$ is the bulk density of ice.

\section{Parameterization impact}

\section{a. Sensitivity studies}

It is important to know how sensitive models are, not only to different parameterization schemes, but also to uncertainties within parameterizations themselves. Different realizations of the relationships between $g$ and $\omega_{0}$ with $r_{e}$ can be generated using coefficients randomly chosen from the uncertainty surfaces representing the parameterization coefficients. There are additional sources of uncertainty not included in the calculations of these surfaces. Some of the more important sources of uncertainty may be errors due to the idealized models that are used to characterize the crystal shapes, due to assumptions on the numbers and masses of small crystals present, and due to uncertainties in the detection and measurement of all-sized ice crystals. M99 showed that details of reflectances, computed using a Monte Carlo radiative transfer code, were somewhat insensitive to the exact details of the classification scheme used, provided the same set of idealized crystals were used in it. The methods used to calculate the radiative properties of the idealized crystals may also affect the results.

Figure 8 shows different realizations of the $g-r_{e}$ re- 


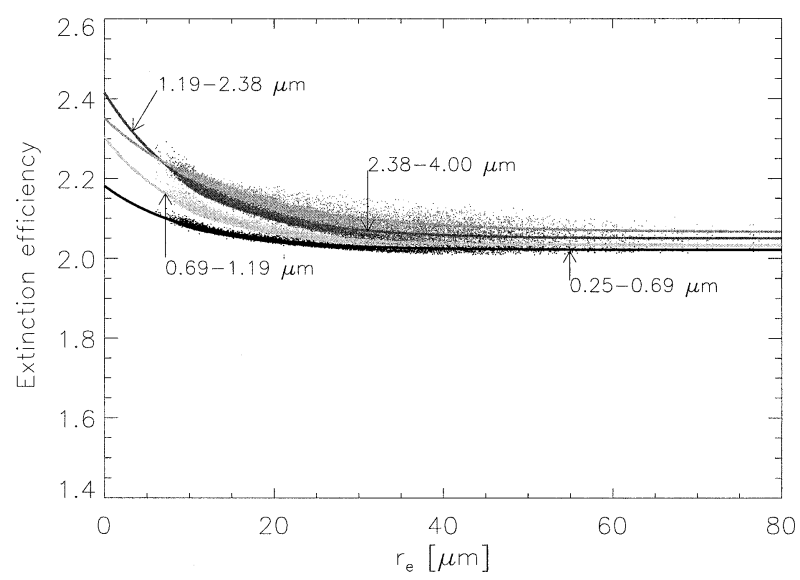

FIG. 7. As in Fig. 5, except for extinction efficiency as a function of $r_{e}$.

lations determined by randomly choosing different coefficients from Table 4. Similar trends are noted for all curves for the same $\lambda$, regardless of which coefficients are randomly chosen from the surface. For most bands, there is a scatter in $g$ values of up to approximately 0.02 depending on $r_{e}$. There is even greater uncertainty in $g$ for small $r_{e}$ for bands 3 and 4. By comparing with Vogelman and Ackerman's (1995) study, it is seen that these uncertainties may be just high enough to impact radiative fluxes up to their flux criterion of $\pm 5 \%$, especially for larger optical depths. By choosing larger or smaller values for $\Delta \chi^{2}$ in deriving the surface of coefficients in parameter phase space, this spread could be either increased or reduced accordingly. It will be seen in the subsequent section that the amount of uncertainty in the $g-r_{e}$ parameterization from the scatter of data is small compared to differences that exist between different parameterization schemes.

Figure 9 shows different realizations of the $\omega_{0}-r_{e}$ relation for the third and fourth bands. The potential variations of $\omega_{0}$ are larger than those for $g$, with spreads of up to 0.03 around a specific $r_{e}$; of course there was little spread for the smallest two wavelengths as the values are nearly unity. The reasons for the larger spread of $\omega_{0}$ values compared to that of the $g$ values are related to the larger spread of the calculated $\omega_{0}$ values in Fig. 6 to that of the $g$ values in Fig. 5. The spread of $Q_{\text {ext }}$ values ranges from 0.02 for bands 1 and 2 at large $r_{e}$, to over 0.2 for bands 3 and 4 at small $r_{e}$ (figures not shown).

There are other uncertainties in the above relations that are not accounted for in the variation of the fit coefficients. One such uncertainty is the representation of the shapes of small ice crystals. Figure 10 compares the $g-r_{e}$ relations predicted using three different shapes to describe the small crystals: the eighth-order Chebyshev polynomials used for the base simulations, the Chebyshev polynomials represented by the CB90 spheroids, and spheres. The differences between the curves are greater than the uncertainties associated with the 


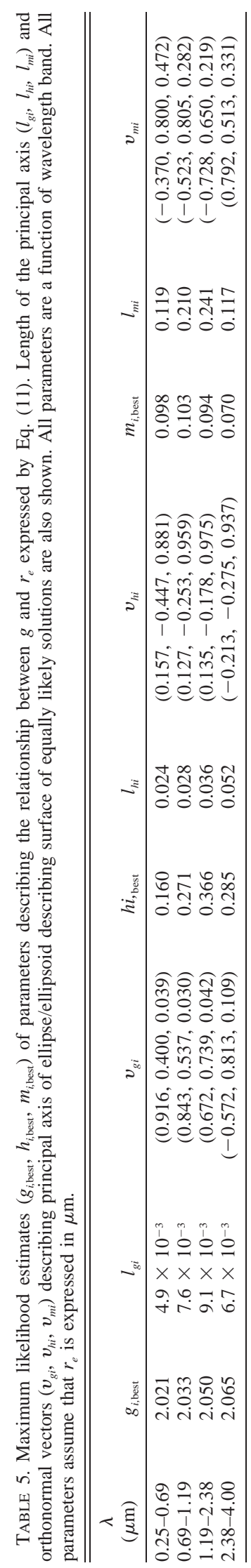

parameterization coefficients (see Fig. 8), showing sensitivity to the assumed shapes of the small crystals. For example, $g$ can differ by up to 0.06 for the different curves for the lowest two $\lambda$ bands; differences are not as substantial for the higher $\lambda$ bands, especially for $2.38-4.0 \mu \mathrm{m}$.

Other uncertainties may also affect the calculations of the library of single scattering properties for small ice crystals. For example, using nonzero sixth-order terms, rather than nonzero eighth-order terms, in the expansion of the Chebyshev polynomials can cause $g$ to deviate by about $2 \%$, but has a smaller impact on $\omega_{0}$. Another sensitivity study could involve the representation of small crystal radiative properties by M96's Koch fractal crystals, as the roughness of these crystals may better model the imperfectness of a crystal's surface. Preliminary calculations show that representing small crystals as Koch fractals can cause substantial differences in the radiative properties of small crystals; a further examination of these differences is beyond the scope of this paper.

Figure 10 also shows two other curves that describe changes in the parameterizations caused by minimizing or maximizing the possible numbers of small crystals according to possible observations. Some of the curves are not visible for some $\lambda$ because they are directly underneath the solid line. The curve that limits the mass of the small crystals does not substantially differ from the base parameterization because only $10 \%$ of the points in the sample had such large mass contents, because changes in $D_{e}$ offset changes in $g$ for such points, and because the numbers of the smallest crystals $(D<$ $20 \mu \mathrm{m})$ did not change significantly under such conditions given the nature of the slope parameterization in Eq. (2). The reasons why increasing the numbers of small crystals to that observed by the FSSP does not impact the parameterization is a little more complex. For bands 1 and 2, the $g$ values calculated for the smaller Chebyshev crystals are not that dissimilar from those parameterized for the merged crystal distributions for the same $r_{e}$. But, even when representing the radiative properties of the smaller crystals with Mie theory, whose $g$ values are larger than those typical for the merged distributions, the parameterized $g$ values obtained from using the FSSP differ only a little from those of the basic merged distributions (figure not shown). This shows that whether the small crystals are represented by measurements from the VIPS or FSSP does not drastically influence the radiative parameterizations describing $\mathrm{g}$, possibly due to a saturation effect that occurs when the numbers of small crystals increases beyond some threshold.

Figure 11 compares the $\omega_{0}-r_{e}$ relations predicted using the three small crystal shapes and variations in numbers of small crystals. The assumed shape of the small crystals is slightly less important than uncertainty in parameterization coefficients (Fig. 9) in varying the $\omega_{0}$ $r_{e}$ relation. The small crystal shape does not have as 

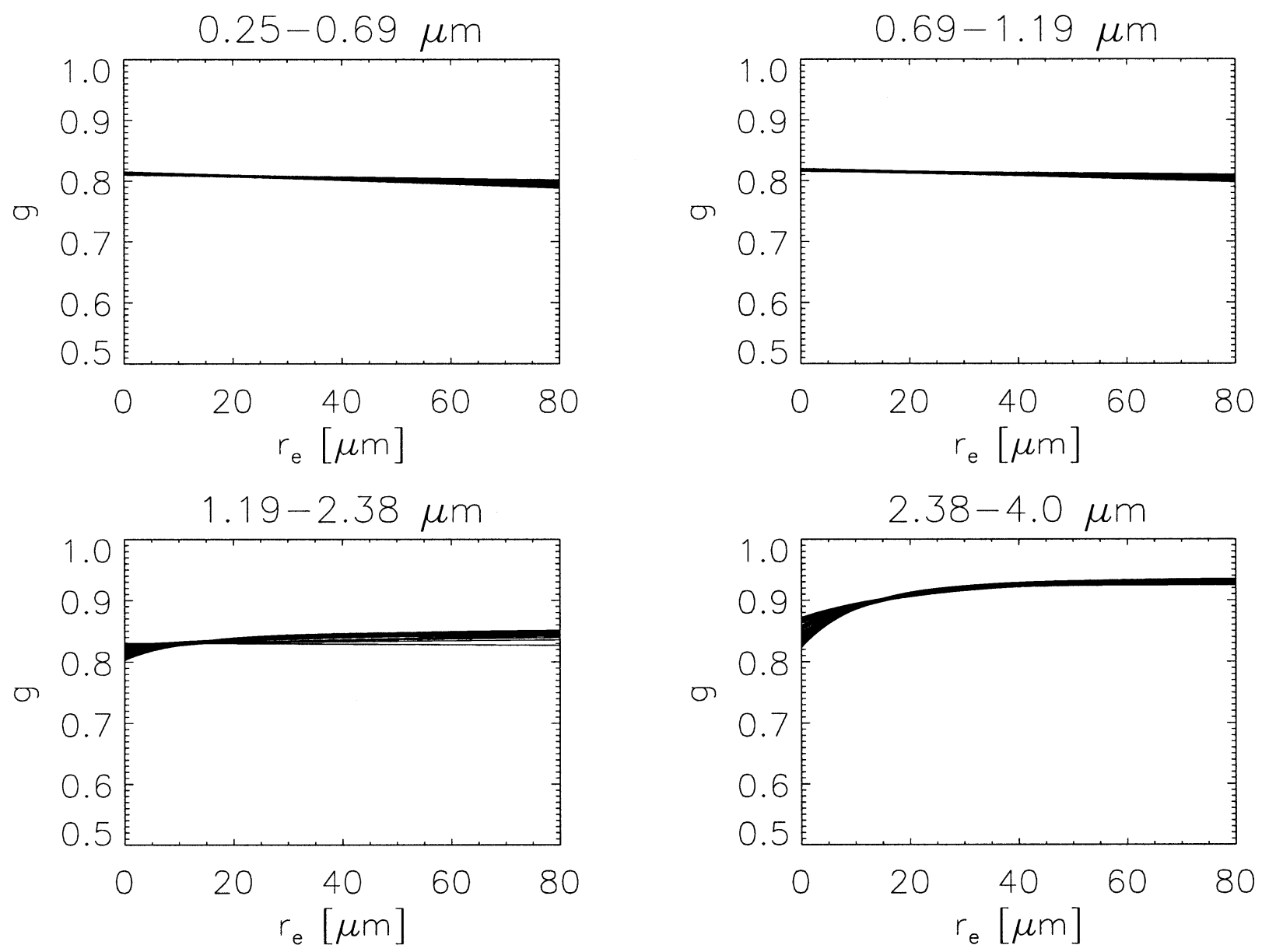

FIG. 8. Asymmetry parameter as a function of $r_{e}$ obtained by parameterization. Different lines represent parameterizations obtained by randomly choosing different $a_{i}, b_{i}$, and $c_{i}$ coefficients from the surface of possible values.
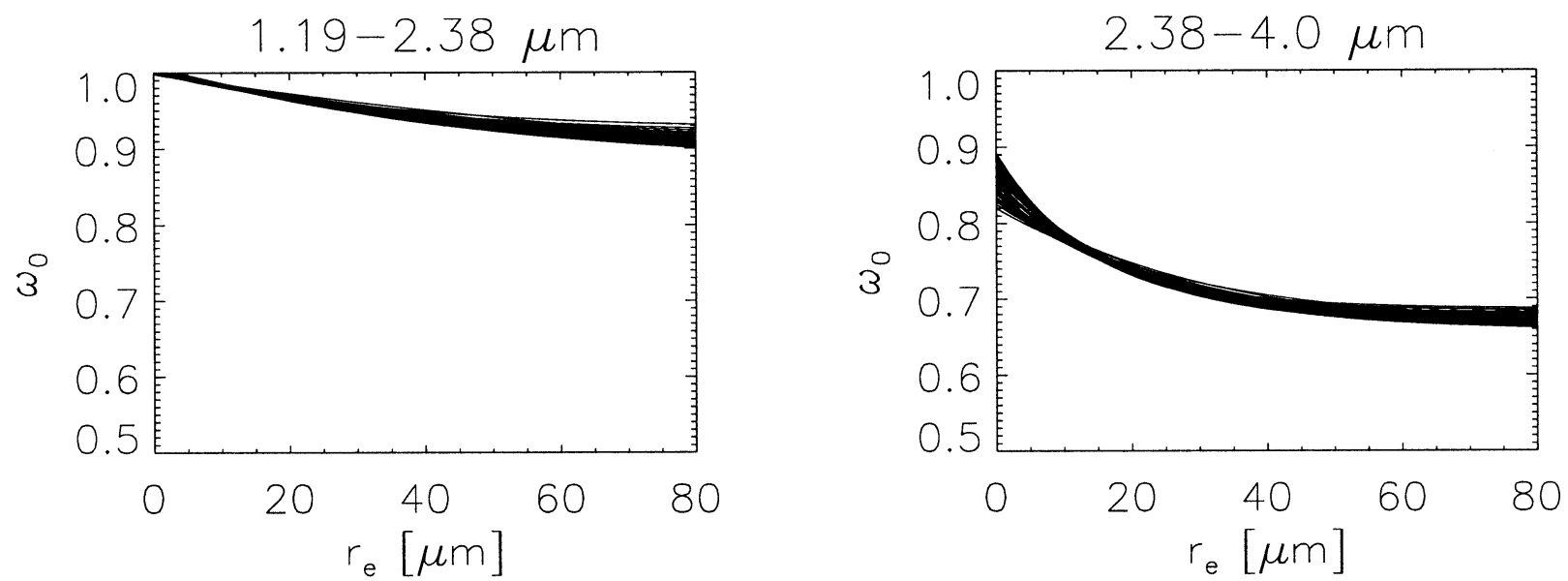

FIG. 9. As in Fig. 8, except different realizations of the $\omega_{0}-r_{e}$ parameterization shown, as obtained by randomly choosing different $d_{i}, e_{i}$, and $f_{i}$ coefficients from the surface of possible values. 

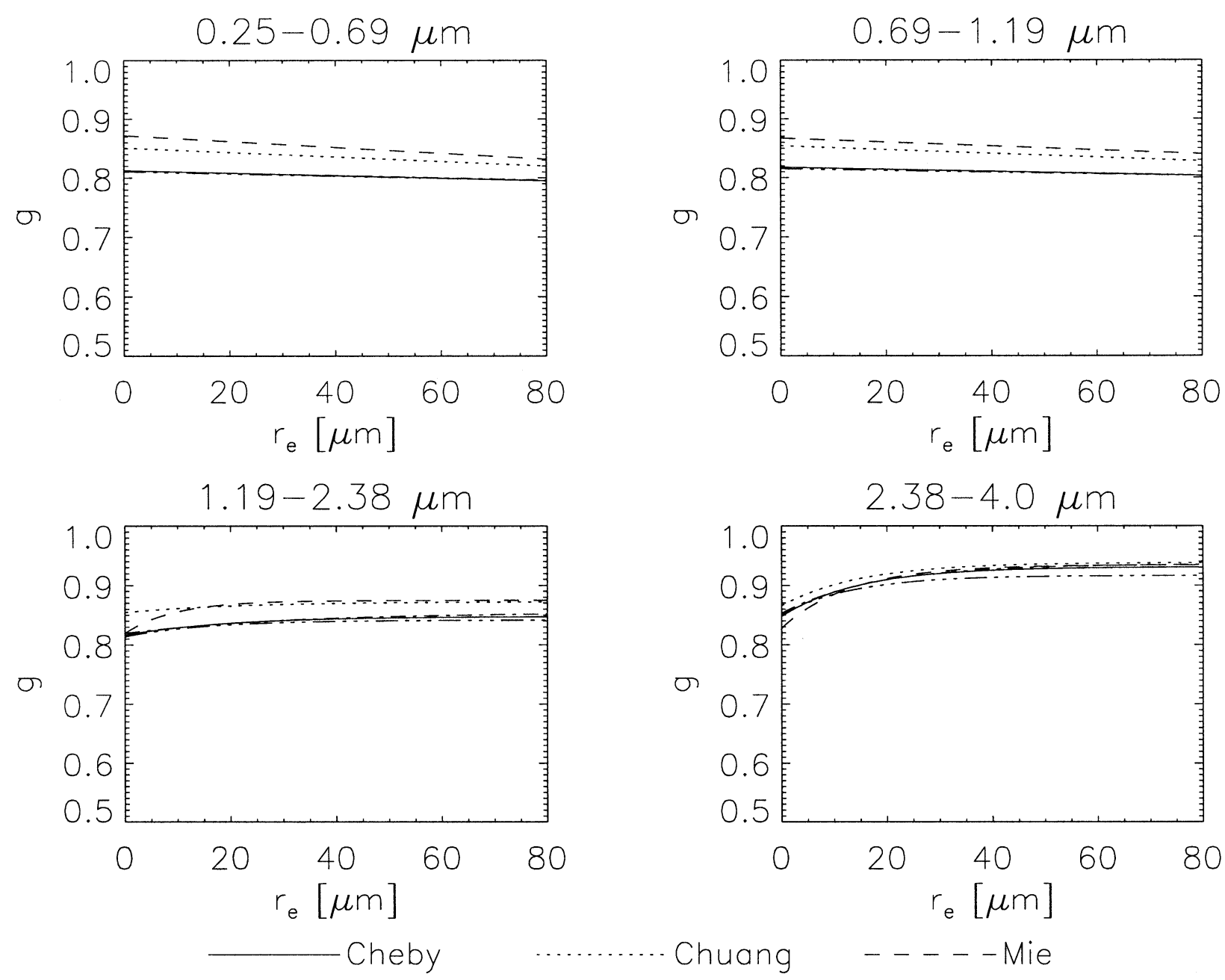

$$
\text { -...-... IWC } \mathrm{S}_{\mathrm{s}} \text { reduced }
$$

$-\cdots-\cdots$. FSSP

FIG. 10. Comparison of $g-r_{e}$ parameterizations obtained by representing the deformed spheres by different shapes of ice crystals: eighthorder Chebyshev polynomials (Cheby), Chuang-Beard 10th-order expansion of Chebyshev polynomials (Chuang), and spheres with radiative properties determined by Mie theory. Dash-dot curve represents limiting the mass of small ice crystals when total IWC exceeds $0.1 \mathrm{~g} \mathrm{~m}^{-3}$, and FSSP represents parameterization obtained assuming that the FSSP can adequately measure small crystals.

large of an impact on the $1.19-2.38-\mu \mathrm{m}$ band as on the $2.38-4.0-\mu \mathrm{m}$ band, with $\omega_{0}$ differing by at most 0.02 for the different small crystal shapes. WY98 previously noted that parameterization schemes for $\omega_{0}$ are not very sensitive to crystal habit when pristine shapes, such as bullet rosettes, columns, aggregates, or needles are considered. The assumed number of small crystals is a bit more important than the assumed shape, especially for the 2.38-4.0- $\mu \mathrm{m}$ band.

Additional sensitivity studies are conducted where it is assumed that all the measured ice crystals could be represented by Koch fractal polycrystals, and where it is assumed that the scattering behavior of the measured crystals could be determined using B01's analytic phase function. Figure 12 compares the $g-r_{e}$ parameterization with those calculated using the M96 and B01 scattering behavior. The curves calculated using M96's polycrystals are not exactly equivalent to the other two curves since they are calculated for a narrowband contained within the broadband used to calculate the other two curves. Calculation of scattering properties of the Koch fractal crystals at sufficient resolution to represent the broadband would have been computationally prohibitive. The differences between the various curves are greater than the uncertainties associated with the parameterization and also greater than the uncertainties associated with the representations of the shapes of small crystals. The larger difference of the M96 curve from the other two curves may be associated with the differences in wavelengths. Similar comparisons for the $g-r_{e}$ relations for the other broadbands showed similar differences between the $g$ values. 


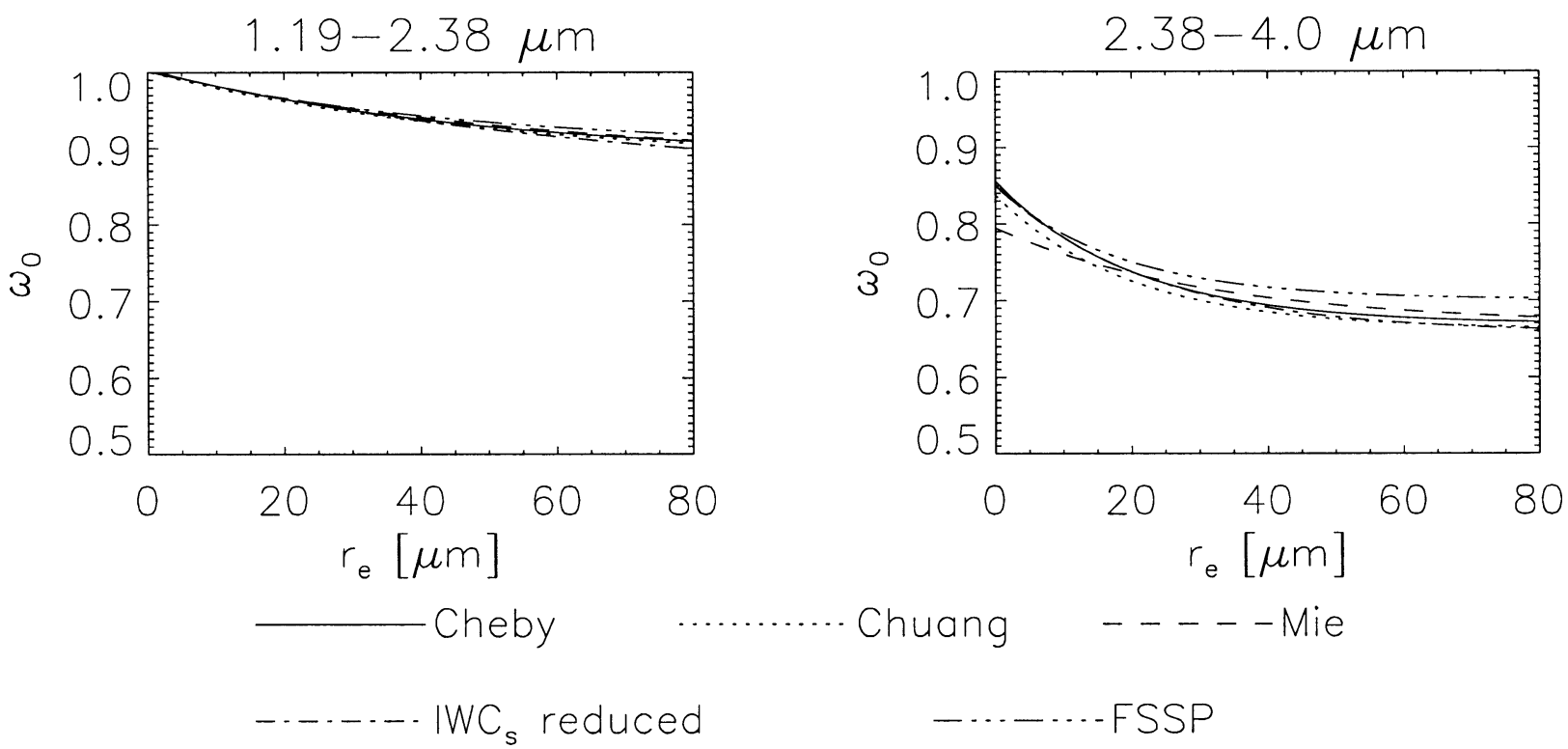

FIG. 11. As in Fig. 10, except comparing $\omega_{0}-r_{e}$ parameterizations for differing small crystal shapes and numbers.

Figure 13 shows comparisons of the $\omega_{0}-r_{e}$ relations for the same radiative libraries. Differences between our parameterization and that based upon the crystal aggregate model of B01 are of similar orders as uncertainties associated with the parameterization coefficients and the representation of small crystal shapes. There are larger differences from calculations made using M96 polycrystals, possibly because of wavelength differences.

\section{b. Comparisons with previous parameterizations}

To better understand the behavior of the parameterization, it is first compared against the single scattering properties of the different sizes and shapes of ice crystals that were used to develop the parameterizations. Figure 14 shows how $g$ varies as a function of $r_{e}$ for the parameterization and for different shapes of crystals. The $r_{e}$ for the aggregates, columns, and bullet rosettes have been adjusted by a factor of $\sqrt{3}$ to account for the difference in $r$ e definitions between this study and that of Y00. For smaller $r_{e}$, especially those between 5 and $30 \mu \mathrm{m}$, the parameterized $g$ closely follows the asymmetry parameters predicted for the deformed spheres due to the large influence of small crystals for small $r_{e}$. For $r_{e}$ smaller than $5 \mu \mathrm{m}$, the parameterized $g$ are higher than the $g$ predicted for the idealized shapes, especially the pristine crystals. The use of the exponential function for bands 3 and 4 somewhat captures this behavior, which is not as prominent for the parameterization due to contributions from smaller crystals. The use of this parameterization may not be appropriate for cirrus that consist principally of pristine shapes, such as hexagonal columns (e.g., tropical tropopause cirrus). For blowoff anvils, it is uncommon to have a majority of ice crystals with sizes smaller than $5 \mu \mathrm{m}$, and even if they were present, they could not be detected by the VIPS instrument that was used to measure the small crystals (MH96). The curve for the deformed sphere is truncated at $40 \mu \mathrm{m}$ because the VIPS measurements were not used for particles with melted equivalent diameters greater than $100 \mu \mathrm{m}$, which approximately corresponds to an $r_{e}$ of $40 \mu \mathrm{m}$. For larger $r_{e}$, the parameterization is closest to the asymmetry parameters for aggregates, which most accurately represent the larger irregular particles imaged by the optical array probes.

Figure 15 compares the new parameterization to previous parameterizations that have been used in climate modeling studies, namely those of EC92, F96, WY98, and K99. All schemes plotted are based on $r_{e}$ defined using Eq. (6), except that of EC92, which used an entirely different definition. For their schemes, EC92 and F96 assumed that ice crystals had the shapes of hexagonal columns, whereas both WY98 and K99 developed various schemes for different shapes of ice crystals. The planar polycrystal scheme of K99 is used because they chose that as their basic crystal shape in their simulations (Kristjansson et al. 2000) and further, this shape most closely matches the irregular shapes of the larger ice crystals observed during CEPEX. The aggregate and bullet rosette parameterizations from WY98 are plotted because these shapes match the observed ice crystals most closely. The WY98 curves are cutoff at various $r_{e}$ in order not to extend their parameterization outside of the range for which it was originally developed and to avoid an unintended decrease of $g$ with $r_{e}$. Because the $r_{e}$ definition used here differs from that used by WY98 by a constant factor of (3) ${ }^{1 / 2}$, this cutoff occurs at much smaller values of $r_{e}$ than occurs in the original paper. Note that if single scattering properties were calculated using weighted mixtures of the properties of 

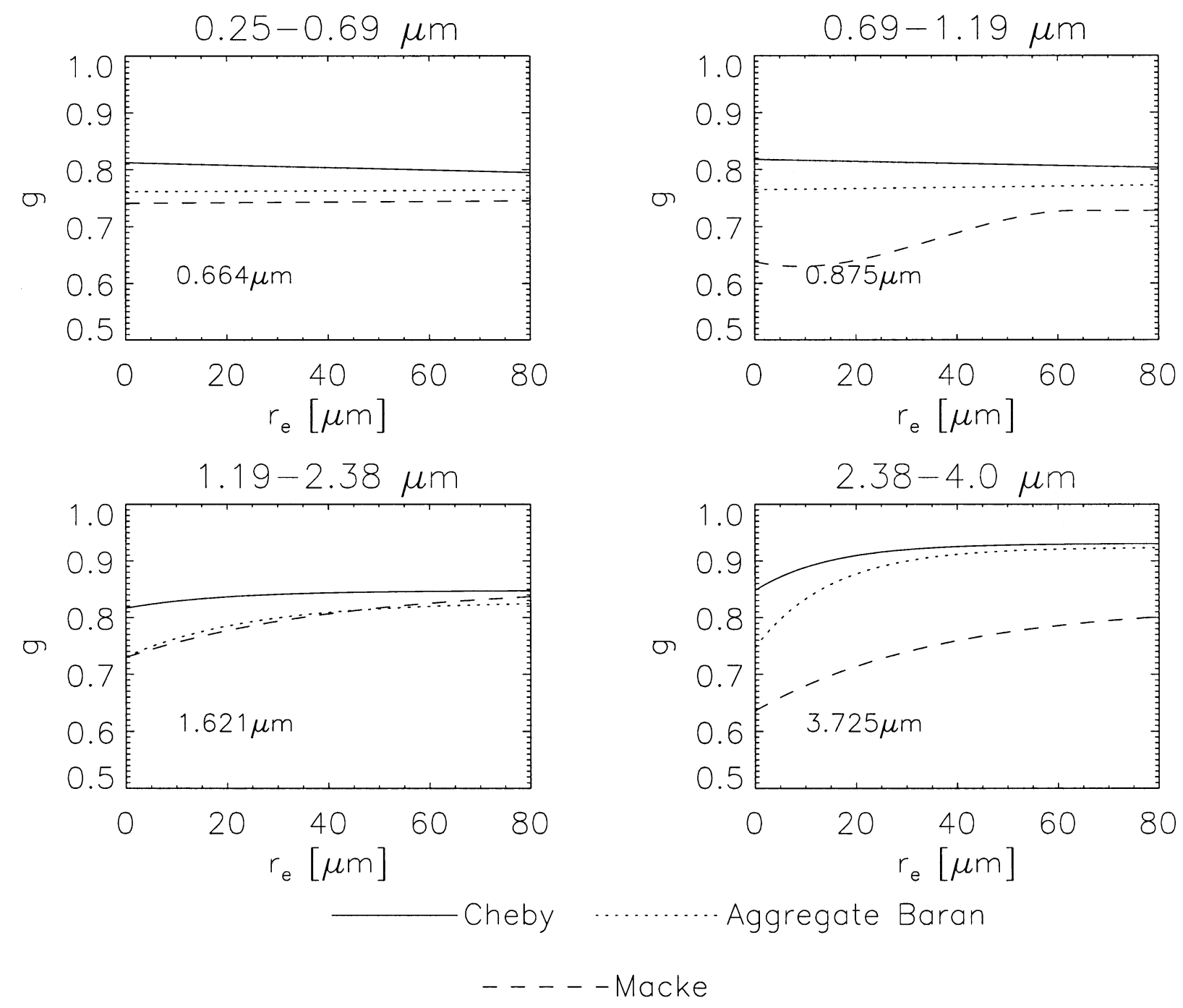

FIG. 12. Comparison of $g-r_{e}$ parameterization developed here, with those determined assuming all crystals scattering properties determined by B01's analytic phase function, and assuming all particles have shapes described by M96's Koch fractal polycrystals. The M96 calculations are for the wavelengths indicated on figure.

WY98 or of K99, better agreement with the new parameterization would probably be seen; differences would only exist due to differences in the calculations of radiative properties.

In Fig. 15, the spread in $g$ is much larger than the spread in $g$ due to different realizations of the parameterization coefficient (Fig. 7) and due to differing representations of small crystals (Fig. 10). This occurs because to the best of our knowledge all of the parameterizations that have previously been used in large-scale models assume single crystal habits; asymmetry parameters depend substantially on crystal shape (e.g., WY98; K99), and different parameterization schemes assume different crystal shapes. The mean parameterization developed here represent an average of the different parameterizations, to some degree, as expected due to the mixture of different crystal habits. The parameterization produced depends crucially on the observed mixture of different shapes and sizes of ice crystals, which can vary substantially depending on day, location of measurements, and cloud type. Thus, the new parameterization depicted represents a mean in a climatological sense for blowoff anvils in the Tropics, from which there can be substantial day-to-day variation.

There can also be differences in the $g-r_{e}$ relations depending on the routines used to calculate the single scattering properties of ice crystals. Figure 16 shows how $g$ varies as a function of $r_{e}$ for distributions of hexagonal columns, as obtained from various radiative transfer calculations. The calculations are for the improved geometric optics technique GOM2 (Y00) for the K99 scheme, which is based on the M96 polycrystal asymmetry parameters (via ray tracing) as reported by Mitchell et al. (1996), the improved geometric ray trac- 

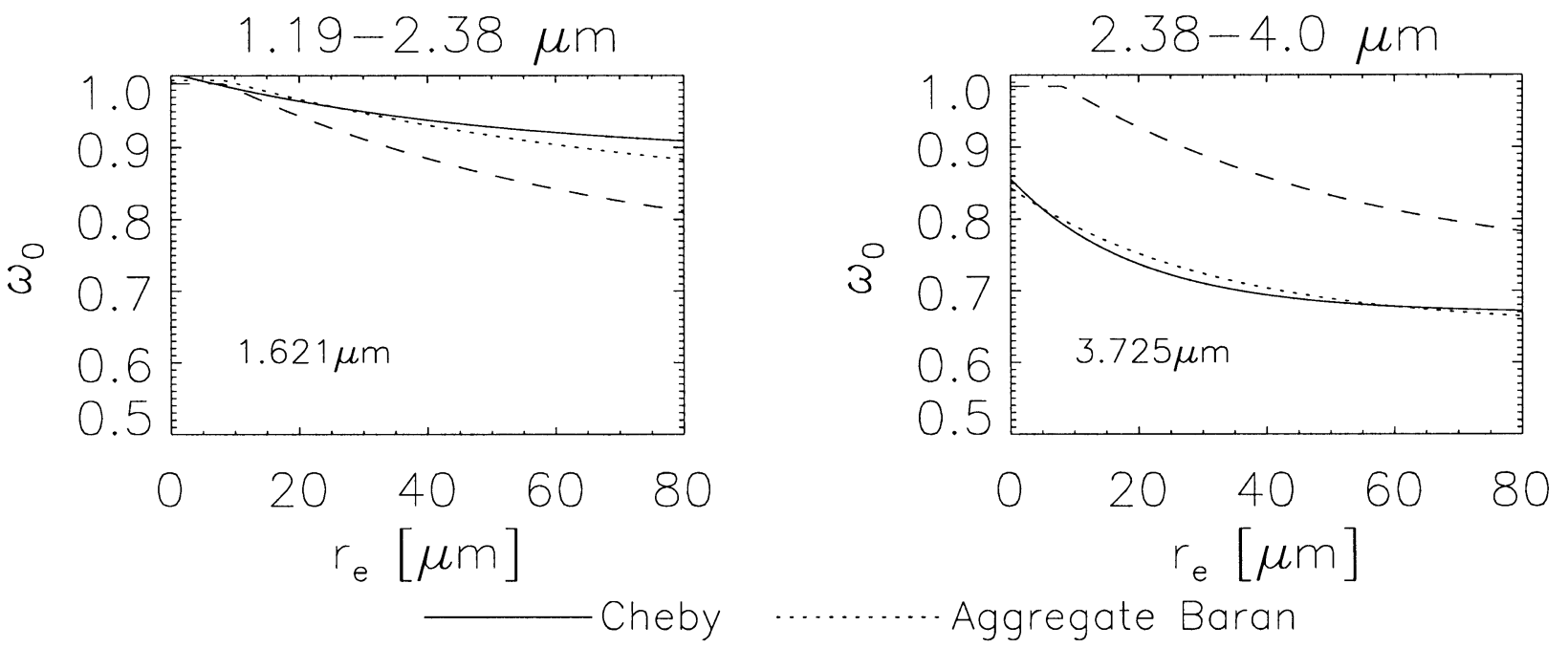

- - - - Macke

FIG. 13. As in Fig. 12, except comparing $\omega_{0}-r_{e}$ parameterizations.

ing program of F96, and EC92's parameterization for hexagonal columns. Note that the uncertainties associated with the use of different radiative transfer algorithms are less than the uncertainties associated with the use of different crystal habits.

Finally, comparisons are made for the $\omega_{0}-r_{e}$ relations that have been used by different investigators. Figure 17 shows how $\omega_{0}$ varies as a function of $r_{e}$ for the parameterization and for the different shapes of ice crystals. As noted by WY98, the $\omega_{0}$ for the pristine shapes to describe the larger crystal sizes depends only weakly on habit; however, the $\omega_{0}$ values for the deformed spheres used to describe the smaller crystals differs substantially from those of the larger crystals. Therefore, the parameterized $\omega_{0}$ asymptotes to values in between those of deformed spheres and these larger crystal shapes.

At first, these discrepancies between $\omega_{0}-r_{e}$ curves for the deformed spheres and pristine crystals seems puzzling, since for constant $r_{e}$, the volume-to-area ratio, which is a rough measure of the photon absorption path, is constant. However, when the size parameter is small, such as for the deformed spheres, the volume-to-area ratio may no longer be a good measure of the photon absorption path and the optical properties may have a strong dependence on the geometry of the particle. This occurs because the approximation made by Bryant and Latimer (1969), namely that the volume-area ratio accounts for phase delay and absorption of rays, is only a good approximation if internal reflection and refraction are ignored. Sun and Fu (2001) recently showed that the Bryant and Latimer (1969) approximation can be in error by as much as $100 \%$ in extinction efficiency when compared against the exact Van de Hulst definition at size parameters around 15 . On the contrary, other studies have suggested that the volume-area ratio is a good benchmark of photon absorption path at all size parameters of interest. More details about the physical reasons for the $\omega_{0}$ differences are not clear at this time since such explanations are not easily extracted from electrodynamic theory.

Figure 18 compares the $\omega_{0}-r_{e}$ relation to previous parameterizations that have been used in climate modeling studies, namely the same schemes depicted in Fig. 15. For the 1.19-2.38- $\mu \mathrm{m}$ band, there are differences between the parameterization schemes, which have a magnitude comparable to the uncertainty in the derived parameterization coefficients, and the newly developed parameterization is almost a mean between other schemes. However, for the 2.38-4.0- $\mu \mathrm{m}$ band, there are substantial differences between the schemes, with the newly developed parameterization giving $\omega_{0}$ values much higher than those from the other schemes. This occurs because deformed spheres, rather than pristine shapes like hexagonal columns, are used to describe the small ice crystals. Note that since only $3.0 \%$ of the solar radiation occurs in the $2.38-4.0-\mu \mathrm{m}$ band, this seemingly large difference in $\omega_{0}$ may not have that large of an impact on modeled solar radiative fluxes.

At this time, it is not easy to conclude which of the above-mentioned parameterization schemes gives the best representation of single scattering radiative properties for use in numerical models. Since ice clouds can consist of very different mixtures of shapes and sizes of ice crystals, it is possible that different schemes are suitable for clouds in different geographical regions or different formation mechanisms (e.g., convective versus nonconvective). The models of B01 and M96 may also be more suitable for some types of clouds than for other types depending on the mixture of ice crystals. Further 

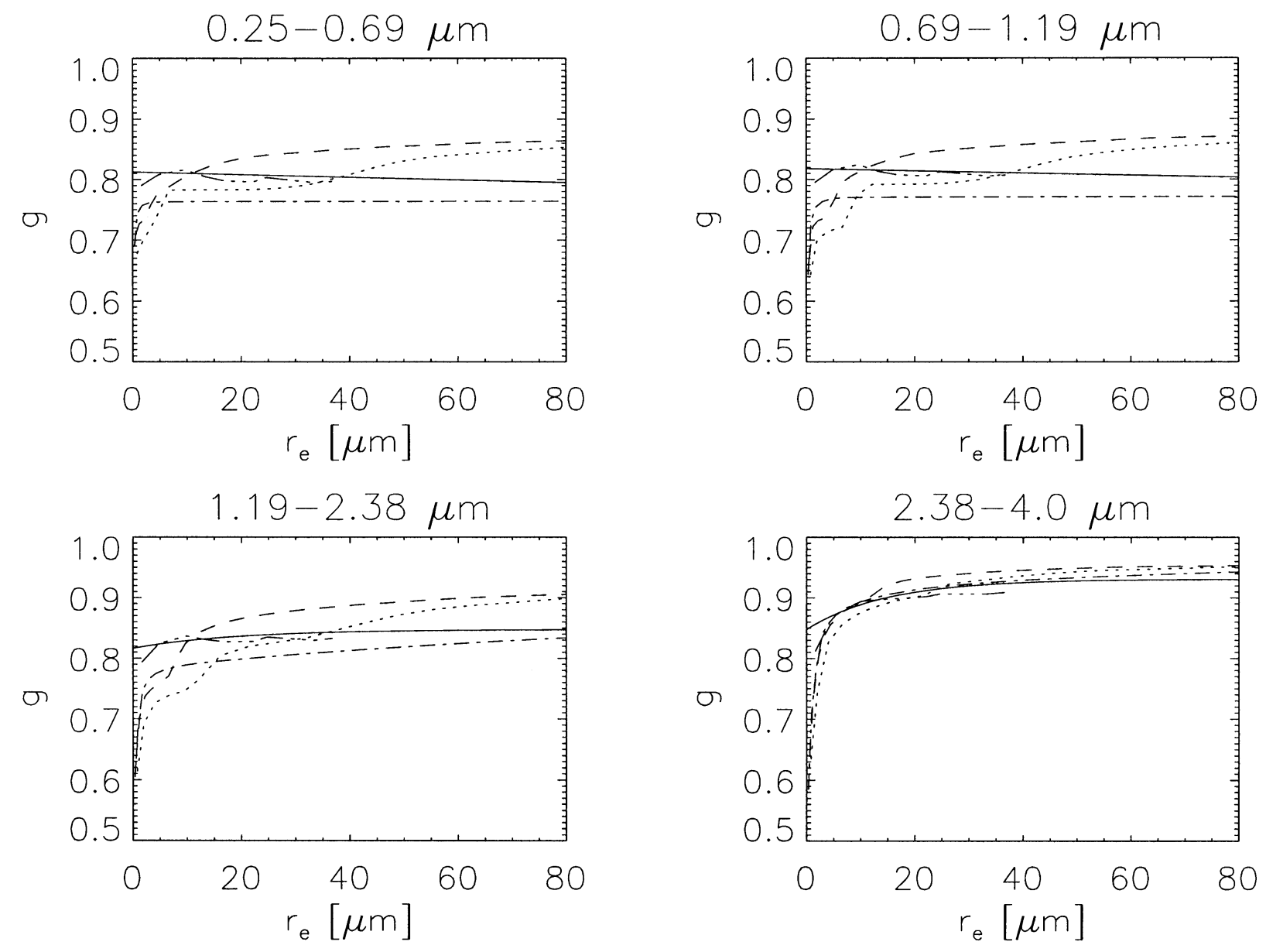

Parameterization Columns -...-Bullet Rosettes

-..-.-Aggregates -...- Deformed spheres

FIG. 14. Asymmetry parameter as function of $r_{e}$. Solid line represents new parameterization developed here, other line types represent relationships for different crystal habits that were used to develop the parameterization.

studies using different mixtures of crystal shapes will help resolve these differences. Comparisons of calculated asymmetry parameters using observed sizes and distributions of ice crystals, with asymmetry parameters directly observed in situ are also required to resolve some of these differences.

\section{Discussion and summary}

New parameterizations of single scattering properties (i.e., $\omega_{0}, g$, and $Q_{\text {ext }}$ ) for distributions of ice crystals observed in tropical anvils have been developed as functions of $r_{e}$ by combining cloud in situ measurements with the results of detailed radiative transfer codes, and are suitable for use in large-scale and cloud resolving models. These parameterizations offer improvements over past parameterizations in that they explicitly account for the characteristics of tropical clouds, are based on observed numbers and shapes of ice crystals smaller than $100 \mu \mathrm{m}$, are based on observed mixtures of different crystal habits, and offer uncertainty estimates in the fit coefficients. The principal findings of this study are summarized below.

1) For a given IWC, there can be substantial scatter in the total mass of small crystals and in the representation of the small crystal size distribution predicted by MH97. This scatter needs to be considered in the calculation of the distributions of microphysical and radiative properties.

2) Because over $90 \%$ of ice crystals smaller than 100 $\mu \mathrm{m}$ were identified as spherical or "quasi-spherical" in shape, their radiative properties are derived from the radiative properties of deformed spheres that are represented by eighth-order Chebyshev polynomials, whose projected area matches the projected area of the observed crystals. These radiative properties differ from those of spherical crystals calculated with Mie theory. 

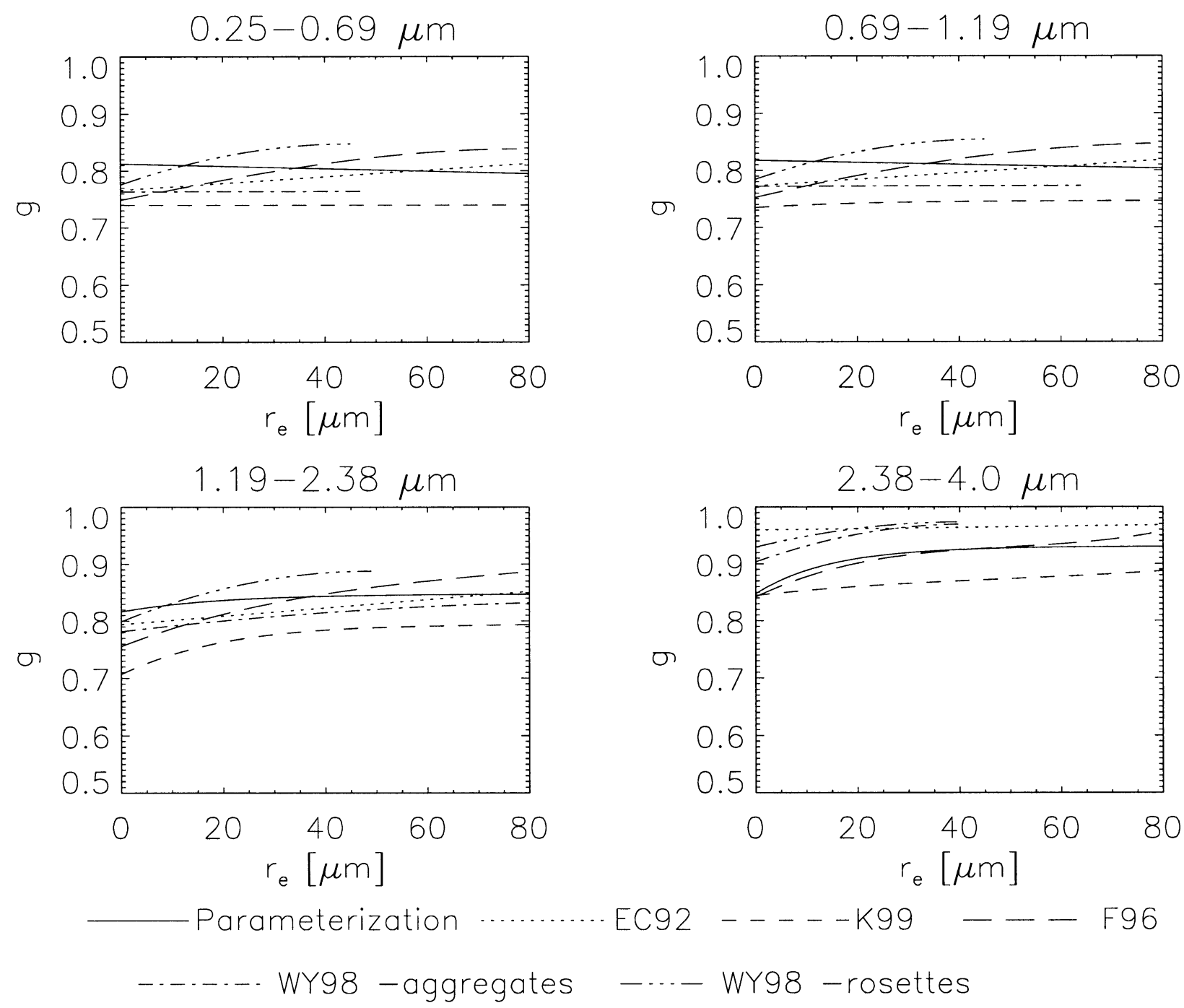

FIG. 15. Comparison of $g-r_{e}$ relationships that have been previously used in large-scale and cloud resolving models. Parameterization developed here represented by solid line.

3) The scattering phase functions, calculated by integrating phase functions for individual ice crystals over distributions of observed mixtures of shapes and sizes of ice crystal, are rather featureless. This replicates the basic structure of other models that have been proposed to describe ice crystal radiative properties based on mixtures of ice crystals.

4) The values of $g, \omega_{0}$, and $Q_{\text {ext }}$ can be parameterized in terms of $r_{e}$ only by either linear or exponential functions depending on $\lambda$. Neither $g, \omega_{0}$, nor $Q_{\text {ext }}$ depend on temperature or IWC in a statistically meaningful manner for the dataset analyzed.

5) Ellipses or ellipsoids in the phase space of fit coefficients are derived from a nonlinear least squared technique to define a surface of possible realizable solutions in fit space. Application of coefficients randomly chosen from this surface shows that there is a possible spread in $g$ of about 0.02 and of $\omega_{0}$ of about 0.04 depending on $\lambda$.

6) Sensitivity studies with different representations of the deformed spheres shows that, although representations affect the calculated radiative properties, the differences are not as large as differences from preexisting parameterizations, or differences associated with choices of different crystal habits. Further, increasing the numbers of small crystals beyond some threshold does not have a substantial effect on the parameterizations of $g$ and $\omega_{0}$.

7) Although $\omega_{0}$ does not depend significantly on the choice of pristine habit for large crystals or on the choice of deformed sphere for small quasi-spherical particles, there are substantial differences in $\omega_{0}$ between the deformed spheres and the large pristine ice crystals. 

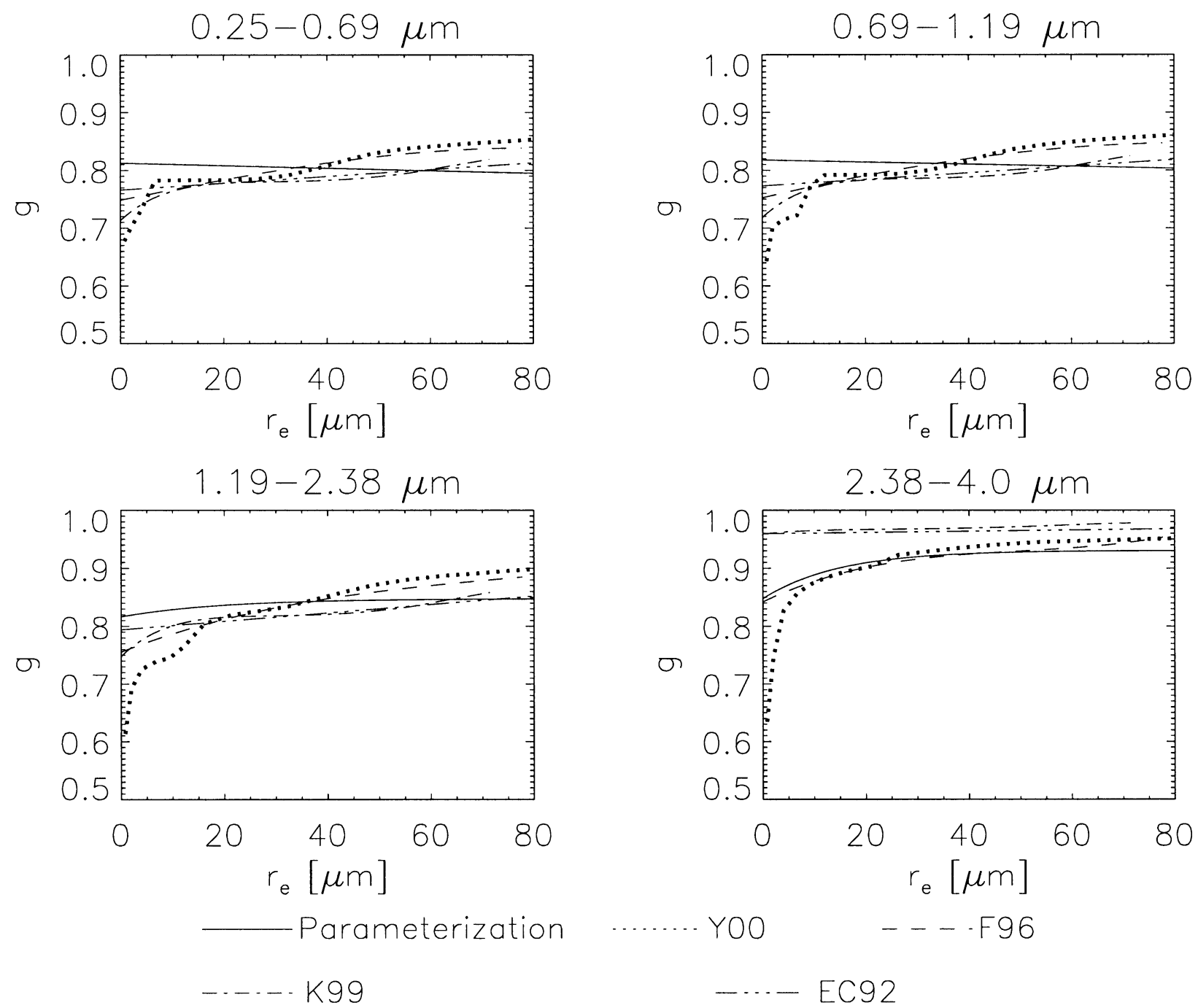

FIG. 16. Comparison of $g-r_{e}$ relationships derived for hexagonal columns using different radiative modeling techniques. Solid line represents the parameterization developed here.

8) There are differences in the parameterizations developed here from other methods designed to account for mixtures of ice crystal shapes and sizes. It cannot be currently resolved which model best represents tropical ice cloud radiative properties.

As with all parameterizations, caution must be taken if it is to be applied under conditions that differ from those under which the original data were collected. Since the data were collected at multiple levels in anvils associated with deep convection in maritime regions, it can be questioned whether the parameterization can be applied at the top of convective updrafts or for large cirrus shields. Only future studies can determine whether these parameterizations can be extended to other cloud systems; additional measurements of the sizes and shapes of small crystals are also sorely needed as well as better idealized habits characterizing crystals observed in situ. Despite this limitation, this study makes an important contribution to microphysical-radiative modeling by using a database larger than those used in previous studies to develop the parameterizations. For example, the EC92 parameterization was based on only four representative ice crystal size distributions obtained in midlatitude cirrus. Future comparisons with angular radiance measurements in the Tropics, might also help resolve some of these issues; although bidirectional measurements are possible with POLDER in the Tropics, rigorous tests of the scattering phase function requires up to 14 different viewing directions, conditions that are only met at midlatitudes and not in the Tropics. Other studies should also concentrate on the development of consistent parameterizations for longwave radiation and on tests using these parameterizations in climate and cloud resolving models.

This study represents an important next step in better defining tropical anvil radiative properties. It also sug- 

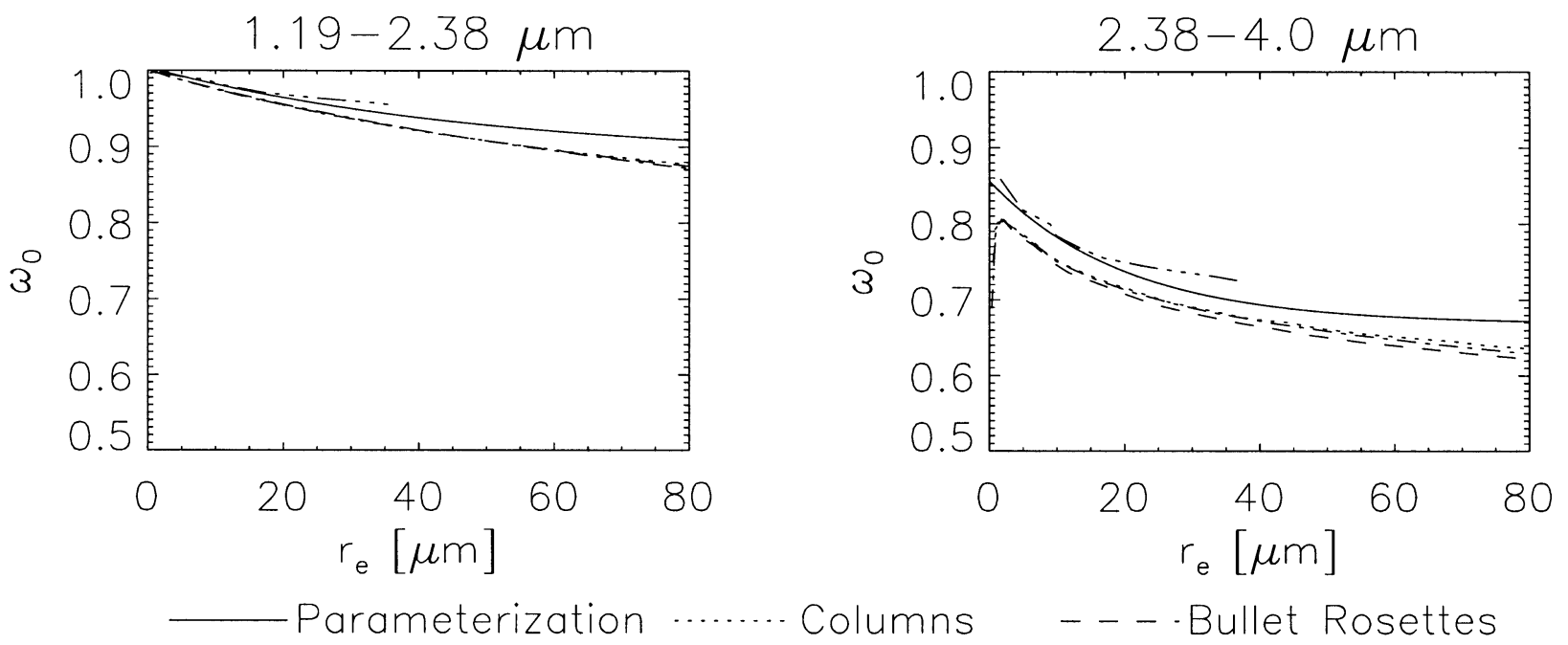

-..-.-Aggregates -...- Deformed spheres

FIG. 17. As in Fig. 14, except for $\omega_{0}-r_{e}$ relationships.

gests that some a priori information about size and habit distributions of ice crystals is required to simulate and retrieve ice cloud properties.

Acknowledgments. This research was supported by the Department of Energy Atmospheric Radiation Measurement (ARM) program under Contract Number DEFG03-00ER62913 and by the National Aeronautics and Space Administration (NASA). Ping Yang's research was supported by the NASA Radiation Sciences Program managed by Dr. Don Anderson. Anthony Baran is supported by the U.K. Department of the Environment, Fisheries and Rural Affairs, under Contract PECD
$7 / 12 / 37$. We are grateful for the assistance and advice of A. Heymsfield, M. Mishchenko, J. Kristjansson, X. $\mathrm{Wu}, \mathrm{R}$. Pincus, and S. Aulenbach. Geoffrey Dix Jr. assisted in manuscript preparation and figure drafting. Substantial comments from two anonymous reviewers and D. Mitchell considerably improved the manuscript. A preliminary version of the parameterization was tested in the Scripps SCM, and we are grateful for the assistance of R. Somerville and S. Iacobellis for the use of this model and their discussions during GMM's visit to Scripps. R. Hobbs, D. Rusk, R. Wallin, and D. Jones were responsible for providing the high-quality microphysical measurements obtained during CEPEX.
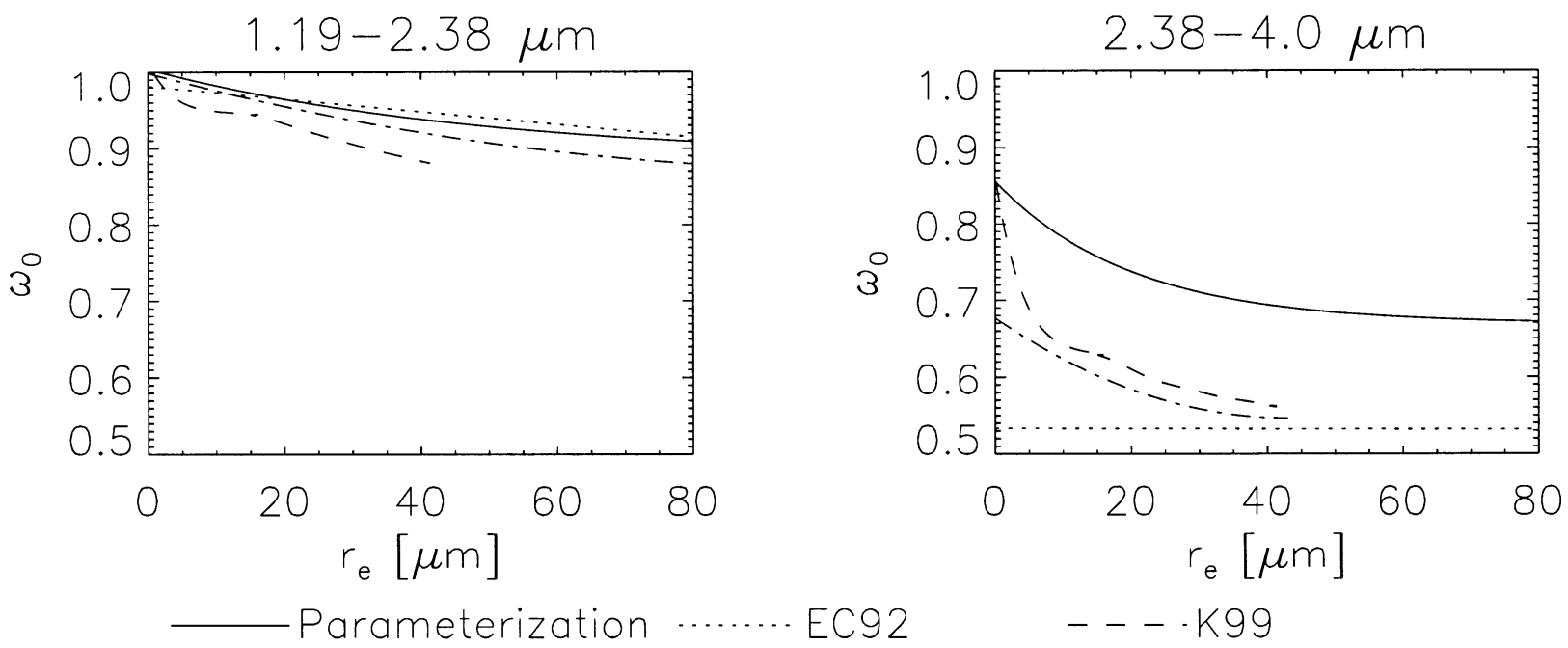


\section{REFERENCES}

Arnott, W. P., D. Mitchell, C. Schmitt, D. Kingsmill, D. Ivanova, and M. R. Poellot, 2000: Analysis of the FSSP performance for measurement of small crystal spectra in cirrus. 13th Int. Conf. on Clouds and Precipitation, Reno, NV, ICCP, 191-193.

Baran, A. J., P. D. Watts, and P. N. Francis, 1999: Testing the coherence of cirrus microphysical and bulk properties from dualviewing multispectral satellite radiance measurements. J. Geophys. Res., 104, 31 673-31 683.

— P. N. Francis, S. Havemann, and P. Yang, 2001a: A study of the absorption and extinction properties of hexagonal ice columns and plates in random and preferred orientation, using exact T-matrix theory and aircraft observations of cirrus. J. Quant. Spectros. Radiat. Transfer, 70, 505-518.

, — L. C.-Labonnote, M. Doutriaux-Boucher, 2001b: A scattering phase function for ice cloud: Tests of applicability using aircraft and satellite multi-angle multi-wavelength radiance measurements of cirrus. Quart. J. Roy. Meteor. Soc., 127, 23952416.

Baumgardner, D., and A. Korolev, 1997: Airspeed corrections for optical array probe sample volumes. J. Atmos. Oceanic Technol., 14, 1224-1229.

Bohren, C. F., and D. R. Huffman, 1983: Absorption and Scattering of Light by Small Particles. Wiley, 544 pp.

Bryant, F. D., and P. Latimer, 1969: Optical efficiencies of large particles of arbitrary shape and orientation. J. Colloid Interface Sci., 30, 291-304.

Chuang, C. C., and K. V. Beard, 1990: A numerical model for the equilibrium shape of electrified raindrops. J. Atmos. Sci., 47, 1374-1389.

Ebert, E. E., and J. A. Curry, 1992: A parameterization of ice cloud optical properties for climate models. J. Geophys. Res., 97, 3831-3836.

Efron, B., and R. J. Tibshirani, 1993: An Introduction to the Bootstrap. Chapman and Hall, 436 pp.

Foot, J. S., 1988: Some observations of the optical properties of clouds. Part II: Cirrus. Quart. J. Roy. Meteor. Soc., 114, 145164.

Francis, P. N., J. S. Foot, and A. J. Baran, 1999: Aircraft measurements of the solar and infrared radiative properties of cirrus and their dependence on ice crystal shape. J. Geophys. Res., 104, 31 685-31695.

Fu, Q., 1996: An accurate parameterization of the solar radiative properties of cirrus clouds. J. Climate, 9, 2058-2082.

Gardiner, B. A., and J. Hallett, 1985: Degradation of in-cloud forward scattering spectrometer probe measurements in the presence of ice particles. J. Atmos. Oceanic Technol., 2, 171-180.

Knollenberg, R. G., K. Kelly, and J. C. Wilson, 1993: Measurements of high number densities of ice crystals in the tops of tropical cumulonimbus. J. Geophys. Res., 98, 8639-8664.

Kristjansson, J. E., J. M. Edwards, and D. L. Mitchell, 1999: A new parameterization scheme for the optical properties of ice crystals for use in general circulation models of the atmosphere. Phys. Chem. Earth B, 24, 231-236.

,$- \ldots$, and -2000 : Impact of a new scheme for optical properties of ice crystals on climates of two GCMs. J. Geophys. Res., 105, $10063-10079$.
Macke, A., and M. Grossklaus, 1998: Light scattering by nonspherical raindrops: Implications for lidar remote sensing of rainrates. $J$. Quant. Spectros. Radiat. Transfer, 60, 355-363.

__ J. Mueller, and E. Raschke, 1996: Single scattering properties of atmospheric ice crystals. J. Atmos. Sci., 53, 2813-2825.

McFarquhar, G. M., and A. J. Heymsfield, 1996: Microphysical characteristics of three anvils sampled during the Central Equatorial Pacific Experiment. J. Atmos. Sci., 53, 2401-2423.

$\longrightarrow$, and _ 1997: Parameterization of tropical cirrus ice crystal spectra and implications for radiative transfer: Results from CEPEX. J. Atmos. Sci., 54, 2187-2201.

_ and _ 1998: The definition and significance of an effective radius for ice clouds. J. Atmos. Sci., 55, 2039-2052.

, ——, A. Macke, J. Iaquinta, and S. M. Aulenbach, 1999: Use of observed ice crystal sizes and shapes to calculate mean scattering properties and multi-spectral radiances: CEPEX 4 April 1993 case study. J. Geophys. Res., 104, 31 763-31 779.

Mitchell, D. L., A. Macke, and Y. Liu, 1996: Modeling cirrus clouds. Part II: Treatment of radiative properties. J. Atmos. Sci., 53, 2967-2988.

Mugnai, A., and W. J. Wiscombe, 1980: Scattering of radiation by moderately nonspherical particles. J. Atmos. Sci., 37, 1291-1307.

Press, W. H., S. A. Teukolsky, W. T. Vetterling, and B. P. Flannery, 1992: Numerical Recipes in FORTRAN. The Art of Scientific Computing. 2d ed. Cambridge University Press, 963 pp.

Ramaswamy, V., and V. Ramanathan, 1989: Solar absorption by cirrus clouds and the maintenance of the tropical upper troposphere thermal structure. J. Atmos. Sci., 46, 2293-2310.

Sun, W., and Q. Fu, 2001: Anomalous diffraction theory for randomly oriented nonspherical particles: A comparison between original and simplified solutions. J. Quant. Spectros. Radiat. Transfer, 70, 737-747.

Takano, Y., and K.-N. Liou, 1989: Solar radiative transfer in cirrus clouds. Part I: Single-scattering and optical properties of hexagonal ice crystals. J. Atmos. Sci., 46, 3-19.

_, and — 1995: Radiative transfer in cirrus clouds. Part III: Light scattering by irregular ice crystals. J. Atmos. Sci., 52, 20 36.

Vogelman, A. M., and T. P. Ackerman, 1995: Relating cirrus cloud properties to observed fluxes: A critical assessment. J. Atmos. Sci., 52, 4285-4301.

Volkovitskiy, O. A., L. N. Pavlova, and A. G. Petrushin, 1980: Scattering of light by ice crystals. Atmos. Oceanic Phys., 16, 90 102.

Wyser, K., and P. Yang, 1998: Average ice crystal size and bulk singlescattering properties of cirrus clouds. Atmos. Res., 49, 315-335.

Yang, P., K. N. Liou, K. Wyser, and D. Mitchell, 2000: Parameterization of the scattering and absorption properties of individual ice crystals. J. Geophys. Res., 105, 4699-4718.

, and Coauthors, 2001: Sensitivity of cirrus birdirectional reflectance to vertical inhomogeneity of ice crystal habits and size distributions for two Moderate-Resolution Imaging Spectroradiometer (MODIS) bands. J. Geophys. Res., 106, 17 267-17 291.

Zhang, Y., A. Macke, and F. Albers, 1999: Effect of crystal size spectrum and crystal shape on stratiform cirrus radiative forcing. Atmos. Res., 52, 59-75. 